\title{
Carrier-carrier scattering in the gain dynamics of InxGa1-xAs/AlyGa1-yAs diode lasers
}

\author{
Sanders, Gary D; Sun, C.-K.; Golubovic, B.; Fujimoto, J. G.; Stanton, Christopher J
}

Published in:

Physical Review B

Link to article, DOI:

10.1103/PhysRevB.54.8005

Publication date:

1996

Document Version

Publisher's PDF, also known as Version of record

Link back to DTU Orbit

Citation (APA):

Sanders, G. D., Sun, C-K., Golubovic, B., Fujimoto, J. G., \& Stanton, C. J. (1996). Carrier-carrier scattering in the gain dynamics of InxGa1-xAs/AlyGa1-yAs diode lasers. Physical Review B, 54(11), 8005-8020.

https://doi.org/10.1103/PhysRevB.54.8005

\section{General rights}

Copyright and moral rights for the publications made accessible in the public portal are retained by the authors and/or other copyright owners and it is a condition of accessing publications that users recognise and abide by the legal requirements associated with these rights.

- Users may download and print one copy of any publication from the public portal for the purpose of private study or research.

- You may not further distribute the material or use it for any profit-making activity or commercial gain

- You may freely distribute the URL identifying the publication in the public portal 


\title{
Carrier-carrier scattering in the gain dynamics of $\operatorname{In}_{x} \mathrm{Ga}_{1-x} \mathrm{As} / \mathrm{Al}_{y} \mathrm{Ga}_{1-y} \mathrm{As}$ diode lasers
}

\author{
G. D. Sanders* \\ Mikroelektronik Centret, Danmarks Tekniske Universitet, DK-2800 Lyngby, Denmark \\ C.-K. Sun \\ Department of Electrical and Computer Engineering, University of California at Santa Barbara, Santa Barbara, California 93106 \\ and Department of Electrical Engineering and Computer Science, Research Laboratory of Electronics, \\ Massachusetts Institute of Technology, Cambridge, Massachusetts 02139 \\ B. Golubovic and J. G. Fujimoto \\ Department of Electrical Engineering and Computer Science, Research Laboratory of Electronics, \\ Massachusetts Institute of Technology, Cambridge, Massachusetts 02139 \\ C. J. Stanton* \\ Mikroelektronik Centret, Danmarks Tekniske Universitet, DK-2800 Lyngby, Denmark \\ (Received 10 October 1995; revised manuscript received 3 June 1996)
}

\begin{abstract}
Ultrafast optical nonlinearities in semiconductors play a central role in determining transient amplification and pulse-dependent gain saturation in diode lasers. Both carrier-phonon and carrier-carrier scattering are expected to determine the gain dynamics in these systems. We present a relaxation-time approximation model for carrier-carrier scattering in strained-layer lasers. The carrier-carrier scattering rates are determined using the quasiequilibrium distribution functions for a given background carrier density. The distribution function to which the photoexcited distribution relaxes is a Fermi-Dirac function where the chemical potential and temperature are self-consistently chosen so that both particle number and energy are conserved in the carriercarrier scattering process. The relaxation approximation makes the problem an effective one-dimensional problem which can then be solved directly for the carrier distributions using an adaptive Runge-Kutta routine. This procedure is less computationally intensive than a full Monte Carlo simulation. The results show that the inclusion of carrier-carrier scattering improves previous results where only carrier-phonon scattering was included and that carrier-carrier scattering is necessary to produce heating of the carriers in the high-energy tails. [S0163-1829(96)07535-2]
\end{abstract}

\section{INTRODUCTION}

Femtosecond pump-probe spectroscopy is a valuable tool for studying the basic physics of nonequilibrium carrier dynamics and high-speed processes in both bulk semiconductors and devices. At the same time, the study of femtosecondgain dynamics in laser diodes plays an important role in understanding laser linewidth, modulation bandwidth, amplification, and short pulse generation. ${ }^{1-6}$ Measurements of ultrafast gain dynamics have been performed in bulk GaAs, ${ }^{7-9}$ bulk $\operatorname{In}_{x} \mathrm{Ga}_{1-x} \mathrm{As}_{1-y} \mathrm{P}_{1-y},{ }^{10} \operatorname{In}_{x} \mathrm{Ga}_{1-x} \mathrm{As}_{\mathrm{y}} \mathrm{P}_{1-y}$ multiple-quantum-well (MQW), ${ }^{11,12}$ and $\operatorname{In}_{x} \mathrm{Ga}_{1-x} \mathrm{As} /$ $\mathrm{In}_{x} \mathrm{Ga}_{1-x} \mathrm{As}_{y} \mathrm{P}_{1-y}$ strained-layer MQW diode amplifiers. ${ }^{13}$

Transient carrier temperature changes, that is, changes in the carrier distribution functions, influence the gain dynamics on 1-ps time scales. These changes in the carrier distribution function can be produced either by free-carrier absorption or by state filling produced by stimulated interband transitions, and two-photon absorption. Initial studies showed that free-carrier absorption can play a dominant role in carrier heating. Later, ${ }^{14}$ it was shown that, not only freecarrier absorption, but stimulated interband transitions contribute to the carrier distribution changes in $\mathrm{In}_{1-x} \mathrm{Ga}_{x} \mathrm{As}_{\mathrm{Al}} / \mathrm{Al}_{1-y} \mathrm{Ga}_{y}$ As strained-layer single-quantum-well (SQW) diode lasers.
In an earlier paper, we presented measurements and calculations of femtosecond gain dynamics in $\mathrm{In}_{1-x} \mathrm{Ga}_{x} \mathrm{As}_{\mathrm{Al}} \mathrm{Al}_{1-y} \mathrm{Ga}_{y} \mathrm{As}$ strained-layer single-quantum-well diode laser. ${ }^{15}$ We presented detailed calculations of gain dynamics in an $\mathrm{In}_{1-x} \mathrm{Ga}_{x} \mathrm{As}_{\mathrm{A}} / \mathrm{Al}_{1-y} \mathrm{Ga}_{y}$ As strained-layer SQW diode laser and compared them to experimental studies. A multiple wavelength femtosecond pump-probe technique was used to study transient gain dynamics in an active diode laser under various injection conditions. We found reasonable agreement between theory and experiment where stimulated emission and absorption were dominant. In our theoretical model, transient gain and differential transmission were computed in a multiband effective-mass model including biaxial strain, valence subband mixing, and polar optical phonon scattering both within and between subbands. Transient photogeneration of electron-hole pairs by the pump pulse and subsequent relaxation of carriers by polar optical phonon scattering were calculated in a Boltzmann equation framework.

In spite of the reasonable agreement between the experimental and theoretical differential transmission curves reported in our earlier paper, improvements can still be made. In our earlier work, the pump and probe wavelengths were fixed and differential transmission curves were measured for several different values of the injection current (i.e., several 


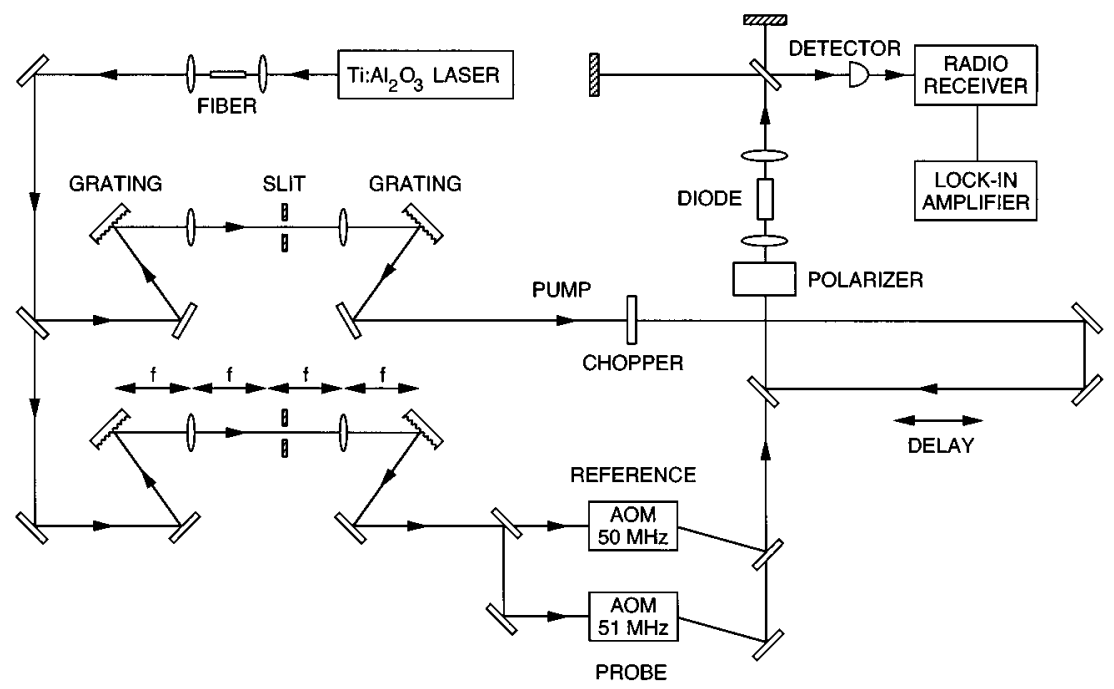

FIG. 1. Heterodyne multiple-wavelength pump-probe measurement technique for measuring time-dependent differential transmission spectra. The pump and probe wavelengths can be varied independently and differential transmission can be studied as a function of probe delay and probe wavelength.

different values of injected carrier concentration). The experimental technique has since been modified to allow for the measurement of time-dependent differential transmission spectra over a range of probe wavelengths (the pump wavelength is still kept fixed). In addition, we extend our theoretical formalism to include carrier-carrier scattering and we compare our results with the experimentally measured differential transmission spectra. Our results show that the inclusion of carrier-carrier scattering improves agreement between experiment and theory where only carrier-phonon scattering was included. Furthermore, carrier-carrier scattering is necessary to produce heating in the high-energy tails of the distribution functions.

Our paper is organized as follows: In Sec. II, we discuss the experimental setup. The theoretical method is presented in Sec. III, and the theoretical results are presented in Sec. IV. The theory is compared to experiment in Sec. V.

\section{EXPERIMENT}

The schematic of the modified experimental setup for the measurement of time-dependent differential transmission ${ }^{16}$ is shown in Fig. 1. The pulse spectrum of a modelocked $\mathrm{TiAl}_{2} \mathrm{O}_{3}$ laser was first broadened in an optical fiber using self-phase modulation and then selected for the pump and the probe using spectral windows. A portion of the probe was split to provide a reference pulse and both probe and reference pulses were frequency shifted using acousto-optic modulators with 50 and $51 \mathrm{MHz}$ modulation frequency, respectively. The pump, probe and reference pulses were coupled collinearly into the diode laser with the reference pulse $1 \mathrm{~ns}$ ahead of the pump and the probe. An imbalanced Michaelson interferometer was used after the device to delay the reference pulse and interfere it with the probe pulse at the detector. An am radio receiver was used to detect the probe signal by monitoring the $1-\mathrm{MHz}$ beat frequency. Background-free measurements were performed by chopping the pump at $400 \mathrm{~Hz}$ and using lock-in detection.

The devices used for these investigations were $\mathrm{In}_{x} \mathrm{Ga}_{1-x} \mathrm{As} / \mathrm{Al}_{y} \mathrm{Ga}_{1-y} \mathrm{As}$ GRIN-SCH SQW diode lasers ${ }^{17}$ which were $300 \mu \mathrm{m}$ in length. The band gap for the active region was near $960 \mathrm{~nm}$ for the heavy-hole transition and near $910 \mathrm{~nm}$ for the light-hole transition. The pump was chosen to be at $935 \mathrm{~nm}$ and the probe wavelengths were varied over a range of $\pm 25 \mathrm{~nm}$ from the pump, both with TE polarizations. The pulse duration was $200 \mathrm{fs}$ with an 8.2-nm bandwidth. Only the $\mathrm{HHl} \rightarrow \mathrm{Cl}$ transition contributes to the initial stimulated emission processes without the light-hole band being directly involved. We performed experiments in the perturbative limit where the differential transmission changes were directly proportional to changes in the gain spectrum.

\section{THEORY}

\section{A. Electronic states and optical-matrix elements}

A schematic of the active region of the strained-layer single-quantum-well diode laser used in the experimental measurements is shown in Fig. 2. It consists of a $100-\AA$ $\mathrm{In}_{0.13} \mathrm{Ga}_{0.87}$ As quantum well surrounded by two $25-\AA \mathrm{GaAs}$ barriers. The $25-\AA$ GaAs barriers, in turn, are sandwiched between two $\mathrm{Al}_{1-x} \mathrm{Ga}_{x} \mathrm{As}$ layers. Because of the lattice mismatch between InAs and GaAs, the quantum well is under biaxial strain. In our treatment of electronic states, we assume that all the strain is accommodated by the $100-\AA$ $\mathrm{In}_{0.13} \mathrm{Ga}_{0.87} \mathrm{As}$ quantum well. We take the growth direction (the direction perpendicular to the heterojunctions) to be

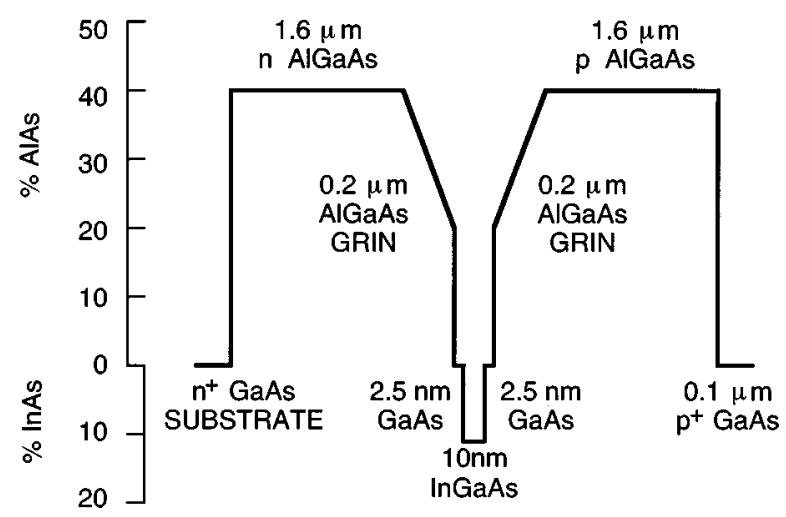

FIG. 2. Schematic structure of $\operatorname{In}_{y} \mathrm{Ga}_{1-y} \mathrm{As} / \mathrm{Al}_{x} \mathrm{Ga}_{1-x} \mathrm{As}$ GRINSCH SQW diode laser. 
along the $z$ direction. Both GaAs and $\mathrm{In}_{1-x} \mathrm{Ga}_{x} \mathrm{As}$ are direct gap materials and we use an effective-mass model to describe the confined electron and heavy- and light-hole states. We solve for the electron and hole envelope functions by finite-differencing the effective-mass Schrödinger equation on an equally spaced mesh. We take the $\mathrm{Al}_{x} \mathrm{Ga}_{x} \mathrm{As}$ barrier to be infinite and require that the effective-mass envelope functions vanish at the $\mathrm{Al}_{1-x} \mathrm{Ga}_{x} \mathrm{As}$ interfaces. The finitedifference equations, together with the vanishing of the envelope functions at the $\mathrm{Al}_{1-x} \mathrm{Ga}_{x} \mathrm{As}$ interfaces, lead to a matrix eigenvalue problem which can be solved to obtain the electron and hole subband energies $E_{n_{c}}(\mathbf{k})$ and $E_{n_{v}}(\mathbf{k})$ as well as the corresponding envelope functions evaluated at the mesh points. Using the computed wave functions, we can obtain the squared optical matrix elements (expressed in units of energy) defined as

$\left|\boldsymbol{\lambda} \cdot \mathbf{P}_{n_{c}, n_{v}}(\mathbf{k})\right|^{2}=\left|\int d \mathbf{r} \psi_{n_{c}, \mathbf{k}}^{*}(\mathbf{r}) \sqrt{2 / m}(\boldsymbol{\lambda} \cdot \mathbf{p}) \psi_{n_{v}, \mathbf{k}}(\mathbf{r})\right|^{2}$.

\section{B. Solution of the Boltzmann transport equation}

To study the time evolution of the distribution functions for electrons or holes in the presence of the pump laser, we use the time-dependent Boltzmann equation,

$$
\frac{\partial f_{n}(\mathbf{k}, t)}{\partial t}=\left[\frac{\partial f_{n}}{\partial t}\right]_{\mathrm{gen}}+\left[\frac{\partial f_{n}}{\partial t}\right]_{\mathrm{sc}}+\left[\frac{\partial f_{n}}{\partial t}\right]_{\mathrm{cc}},
$$

where $f_{n}(\mathbf{k}, t)$ is the time-dependent distribution function for electrons (or holes) in subband, $n$ with wave vector $\mathbf{k}$. The first term on the right-hand side represents the time rate of change of the distribution functions due to transient photogeneration of electron-hole pairs by the pump, while the second term represents the time rate of change due to scattering by polar optical phonons. The final term describes the time rate of change of the distribution functions due to carriercarrier scattering. To simplify the calculations, we adopt an axial approximation in which the distribution functions are replaced by their angular averages in the plane of the quantum well. We define axial distribution functions

$f_{n}(k, t) \equiv \frac{1}{2 \pi} \int_{0}^{2 \pi} d \theta f_{n}(\mathbf{k}, t)=\frac{1}{2 \pi} \int_{0}^{2 \pi} d \theta f_{n}(k, \theta, t)$,

where we have written the wave vector in cylindrical coordinates $\mathbf{k}=k \hat{\rho}+\theta \hat{\theta}$. We take the moment of the Boltzmann equation over $\theta$ to obtain a one-dimensional Boltzmann equation:

$$
\begin{aligned}
\frac{\partial f_{n}(k, t)}{\partial t}= & \frac{1}{2 \pi} \int_{0}^{2 \pi} d \theta\left[\frac{\partial f_{n}(k, t)}{\partial t}\right]_{\mathrm{gen}} \\
& +\frac{1}{2 \pi} \int_{0}^{2 \pi} d \theta\left[\frac{\partial f_{n}(k, t)}{\partial t}\right]_{\mathrm{sc}} \\
& +\frac{1}{2 \pi} \int_{0}^{2 \pi} d \theta\left[\frac{\partial f_{n}(k, t)}{\partial t}\right]_{\mathrm{cc}} .
\end{aligned}
$$

To solve the one-dimensional Boltzmann equations, we convert them to a coupled set of ordinary differential equa- tions (ODE's). In the ODE method, we divide $k$ space into evenly spaced cells of width $\Delta k=k_{\max } / N_{k}$ where $N_{k}$ is the number of $k$ cells and the value of $k$ at the midpoint of each cell is $k_{m}\left(m=1, \ldots, N_{k}\right)$. In each $k$ cell the average of the distribution function is defined as

$$
f_{n}\left(k_{m}, t\right) \equiv \frac{1}{k_{m} \Delta k} \int_{k_{m}-\Delta k / 2}^{k_{m}+\Delta k / 2} d k k f_{n}(k, t) .
$$

Taking the weighted average of the one-dimensional Boltzmann equation over a $k$ cell and assuming the distribution functions are slowly varying in each cell, we obtain

$$
\begin{aligned}
\frac{d f_{n}\left(k_{m}, t\right)}{d t}= & {\left[\frac{d f_{n}\left(k_{m}, t\right)}{d t}\right]_{\mathrm{sc}}+\left[\frac{d f_{n}\left(k_{m}, t\right)}{d t}\right]_{\mathrm{gen}} } \\
& +\left[\frac{d f_{n}\left(k_{m}, t\right)}{d t}\right]_{\mathrm{cc}} .
\end{aligned}
$$

This is a set of ordinary differential equations for $f_{n}\left(k_{m}, t\right)$.

Given initial values of the distribution functions in each $k$ cell, $f_{n}\left(k_{m}, t \rightarrow-\infty\right)$, we can solve the system of ODE's with an adaptive stepsize Runge-Kutta routine. The initial distribution functions are determined by the electron and hole quasi-Fermi levels. The initial electron distribution functions are

$$
f_{n_{c}}\left(k_{m}, t \rightarrow-\infty\right)=\frac{1}{1+\exp \left\{\left[E_{n_{c}}\left(k_{m}\right)-F_{c}\right] / k_{B} T\right\}},
$$

while the initial hole distribution functions are given by

$$
f_{n_{v}}\left(k_{m}, t \rightarrow-\infty\right)=\frac{1}{1+\exp \left\{-\left[E_{n_{v}}\left(k_{m}\right)-F_{v}\right] / k_{B} T\right\}} .
$$

Here we use the angular averaged subbands

$$
E_{n}(k)=\frac{1}{2 \pi} \int_{0}^{2 \pi} d \theta E_{n}(\mathbf{k}) .
$$

The electron and hole quasi-Fermi levels, $F_{c}$ and $F_{v}$, are related to the total carrier concentration (assumed equal for electrons and holes) by

$$
\begin{aligned}
n_{2 \mathrm{D}} & =\frac{1}{\pi} \sum_{n_{c}, m} f_{n_{c}}\left(k_{m}, t \rightarrow-\infty\right) k_{m} \Delta k \\
& =\frac{1}{\pi} \sum_{n_{v}, m} f_{n_{v}}\left(k_{m}, t \rightarrow-\infty\right) k_{m} \Delta k .
\end{aligned}
$$

A factor of 2 has been included to account for the spin of electrons and holes.

For the electron photogeneration term, we have

$$
\begin{aligned}
{\left[\frac{\partial f_{n_{c}}\left(k_{m}, t\right)}{\partial t}\right]_{\text {gen }} } & \propto \sum_{n_{v}} u(\hbar \omega, t)\left|\boldsymbol{\lambda} \cdot \mathbf{P}_{n_{c}, n_{v}}\left(k_{m}\right)\right|^{2} \\
& \times\left[1-f_{n_{c}}\left(k_{m}, t\right) \mid-f_{n_{v}}\left(k_{m}, t\right)\right],
\end{aligned}
$$

where the photon energy is

$$
\hbar \omega=E_{n_{c}}\left(k_{m}\right)-E_{n_{v}}\left(k_{m}\right)
$$


and the transient energy density of the pump is an ideal Gaussian pulse,

$$
\begin{aligned}
u(\hbar \omega, t) \propto \operatorname{sech}^{2}\left(\frac{2 t \cosh ^{-1}(\sqrt{2})}{\tau_{0}}\right) & \\
& \times \exp \left(\frac{-4 \ln (2)}{\sigma^{2}}\left(\hbar \omega-\hbar \omega_{0}\right)^{2}\right) .
\end{aligned}
$$

The pulse is centered at $\hbar \omega_{0}$ with a temporal width $\tau_{0}$ and a spectral width $\sigma$. It is worth noting that the photogeneration term at a given $k$ can be positive or negative depending on the sign of $1-f_{n_{c}}\left(k_{m}, t\right)-f_{n_{v}}\left(k_{m}, t\right)$. This method of generating carriers is quasiclassical and is valid providing the dephasing time of the photoexcited carriers is short with respect to the pulse duration; otherwise carriers must be generated using the semiconductor Bloch equations. In generating the photoexcited carriers, we first use the full (nonangular averaged) bands to determine an "effective pulse width" that is then used to generate carriers in the angular averaged bands.

The first two terms in the Boltzmann equation (6) describing photogeneration and scattering by polar optical phonons have been described in detail in our earlier paper. ${ }^{15}$ Here we focus on the carrier-carrier scattering term. We model carrier-carrier scattering using an effective relaxation operator approach. ${ }^{18}$ Carrier-carrier scattering for both electrons and holes is described by an effective relaxation operator of the form

$$
\left[\frac{\partial f_{n}(k, t)}{\partial t}\right]_{\mathrm{cc}}=-\frac{f_{n}(k, t)-f_{n}^{0}\left(k, \mu^{*}, T^{*}\right)}{\tau_{n}(k)} .
$$

The relaxation time, $\tau_{n}(k)$, depends on subband index $n$ and wave vector $k$ and (in the case of electrons)

$$
f_{n}^{0}\left(k, \mu^{*}, T^{*}\right)=\frac{1}{1+\exp \left\{\left[E_{n}(k)-\mu^{*}\right] / k_{B} T^{*}\right\}}
$$

is a quasiequilibrium distribution function characterized by an effective temperature $T^{*}$ and chemical potential $\mu^{*}$. In the usual relaxation-time approximation, we would set $T^{*}$ to the lattice temperature and $\mu^{*}$ to the electron quasi-Fermi level $F_{c}$. In the effective relaxation operator approach, the effective temperature and chemical potential are time dependent. They are determined by the constraints that the particle number and energy are conserved in carrier-carrier collisions. Since $\tau(k)$ is $k$ dependent, the actual distribution function $f_{n}(k, t)$ and the relaxation function $f_{n}^{0}\left(k, \mu^{*}, T^{*}\right)$ may be characterized by different chemical potentials and effective temperatures. They are equal if the actual distribution function is a Fermi-Dirac function as it must be when $t \rightarrow \infty$.

Imposing conservation of mass on the carrier-carrier scattering mechanism we obtain

$$
\frac{\partial}{\partial t} \sum_{n, k} f_{n}(k, t)=\sum_{n, k}\left[\frac{\partial f_{n}(k, t)}{\partial t}\right]_{\mathrm{cc}}=0,
$$

which, in the axial approximation, becomes

$$
\sum_{n} \int_{0}^{\infty} d k k\left[\frac{f_{n}(k, t)-f_{n}^{0}\left(k, \mu^{*}, T^{*}\right)}{\tau_{n}(k)}\right]=0 .
$$

This integral may be evaluated using Simpson's rule. Thus

$$
C_{1}\left(\mu^{*}, T^{*}, t\right)=\sum_{n, m} \Delta k k_{m}\left[\frac{f_{n}\left(k_{m}, t\right)-f_{n}^{0}\left(k, \mu^{*}, T^{*}\right)}{\tau_{n}\left(k_{m}\right)}\right]=0 .
$$

This last equation may be viewed as a global equation of constraint to be satisfied by the effective temperature and chemical potential. From the requirement that carrier-carrier scattering conserve energy, we obtain a second equation of constraint

$$
\begin{aligned}
C_{2}\left(\mu^{*}, T^{*}, t\right)= & \sum_{n, m} \Delta k k_{m} E_{n}\left(k_{m}\right) \\
& \times\left[\frac{f_{n}\left(k_{m}, t\right)-f_{n}^{0}\left(k_{m}, \mu^{*}, T^{*}\right)}{\tau_{n}\left(k_{m}\right)}\right]=0 .
\end{aligned}
$$

The two equations of constraint, $C_{1}\left(\mu^{*}, T^{*}, t\right)=0$ and $C_{2}\left(\mu^{*}, T^{*}, t\right)=0$, uniquely determine the effective temperature and chemical potential at any given time in terms of the time-dependent cell-averaged distribution functions and the subband and wave-vector-dependent relaxation rates. In the Runge-Kutta integration of the Boltzmann equation, we use a Newton-Raphson multidimensional root-finding routine to obtain the effective temperature and chemical potential needed for the evaluation of the effective relaxation operator.

\section{Relaxation rates for electrons}

In order to employ the effective relaxation operator for electron-electron scattering in the Boltzmann transport equation, we still need to compute the relaxation rates, $1 / \tau_{n}\left(k_{m}\right)$, for electrons and holes. In the case of electrons, we assume that the carriers all stay in their original subbands (diagonal approximation). That is, carriers can scatter with other carriers in different bands, but they do not change bands in the scattering process. We let $W_{\mathbf{k}, \mathbf{k}^{\prime}}^{n, n^{\prime}}(\mathbf{q})$ be the two-body scattering rate at which an electron initially in state $|n, \mathbf{k}\rangle$ scatters off a second electron initially in state $\left|n^{\prime}, \mathbf{k}^{\prime}\right\rangle$ and ends up in a final state $|n, \mathbf{k}+\mathbf{q}\rangle$ having scattered the second electron to the state $\left|n^{\prime}, \mathbf{k}^{\prime}-\mathbf{q}\right\rangle$.

Neglecting Fermi filling effects, the two-body scattering rate obtained from Fermi's golden rule is

$$
\begin{aligned}
W_{\mathbf{k}, \mathbf{k}^{\prime}}^{n, n^{\prime}}(\mathbf{q})= & \frac{2 \pi}{\hbar}\left|V_{\mathbf{k}, \mathbf{k}^{\prime}}^{n, n^{\prime}}(\mathbf{q})\right|^{2} \delta\left(E_{n}(|\mathbf{k}+\mathbf{q}|)+E_{n^{\prime}}(|\mathbf{k}-\mathbf{q}|)\right. \\
& \left.-E_{n}(|\mathbf{k}|)-E_{n^{\prime}}\left(\left|\mathbf{k}^{\prime}\right|\right)\right)
\end{aligned}
$$

The two-body interaction matrix is just

$$
\begin{aligned}
V_{\mathbf{k}, \mathbf{k}^{\prime}}^{n, n^{\prime}}(\mathbf{q})= & \int d \mathbf{r} \int d \mathbf{r}^{\prime} \psi_{n, \mathbf{k}}^{*}(\mathbf{r}) \psi_{n^{\prime}, \mathbf{k}^{\prime}}^{*}\left(\mathbf{r}^{\prime}\right) \\
& \times V\left(\left|\mathbf{r}-\mathbf{r}^{\prime}\right|\right) \psi_{n, \mathbf{k}+\mathbf{q}}(\mathbf{r}) \psi_{n^{\prime}, \mathbf{k}^{\prime}-\mathbf{q}}\left(\mathbf{r}^{\prime}\right),
\end{aligned}
$$

where $V\left(\left|\mathbf{r}-\mathbf{r}^{\prime}\right|\right)$ is the screened Coulomb potential. In the effective-mass approximation, the quantum well electron wave function for an electron in the $n$th subband is 


$$
\psi_{n, \mathbf{k}}(\mathbf{r})=\frac{1}{\sqrt{A}} e^{i \mathbf{k} \cdot \mathbf{r}} F_{n}(z)\left|J=\frac{1}{2}, \sigma\right\rangle,
$$

where $F_{n}(z)$ is the electron envelope function described in Ref. 15 and $\left|J=\frac{1}{2}, \sigma\right\rangle$ is the periodic part of the $s$-like $k=0$ Bloch state in the bulk material. For a statically screened Coulomb potential, we obtain ${ }^{19}$

$$
V_{\mathbf{k}, \mathbf{k}^{\prime}}^{n, n^{\prime}}(\mathbf{q})=\frac{2 \pi e^{2}}{A \epsilon_{0} q \epsilon(q)} F_{n, n^{\prime}}(q),
$$

where the form factor

$$
\begin{aligned}
F_{n, n^{\prime}}(q) \equiv & \int_{-\infty}^{\infty} d z \int_{-\infty}^{\infty} d z^{\prime}\left|F_{n}(z)\right|^{2}\left|F_{n^{\prime}}\left(z^{\prime}\right)\right|^{2} \\
& \times \exp \left(-q\left|z-z^{\prime}\right|\right) .
\end{aligned}
$$

It should be pointed out that in the strict two-dimensional limit, $F_{n, n^{\prime}}(q)=1$. Thus $F_{n, n^{\prime}}(q)$ accounts for the finite size of the quantum well. In our treatment of screening, we assume that most of the electrons reside in the lowest-lying electron subband (the electric quantum limit approximation). The dielectric function for electrons in a quantum well is then given in the random phase approximation (RPA) by ${ }^{20}$

$$
\epsilon(q)=\left(1+\frac{q_{s}}{q}\right) F_{1,1}(q)\left\{1-\Theta\left(q-2 k_{F}\right) \sqrt{1-\left(2 k_{F} / q\right)^{2}}\right\},
$$

where $k_{F}=\sqrt{2 \pi n}$ is the Fermi wave vector for carrier concentration, $n$, and

$$
q_{s}=\frac{2 e^{2} m_{e}^{*}}{\epsilon_{0} \hbar^{2}}
$$

is the screening wave vector. The electron effective mass is $m_{e}^{*}$ and $F_{1,1}(q)$ corrects for the finite size of the quantum well. With the RPA dielectric function, the screened Coulomb matrix element is given by the rather formidable looking expression

$$
\begin{aligned}
V_{\mathbf{k}, \mathbf{k}^{\prime}}^{n, n^{\prime}}(\mathbf{q})= & \frac{2 \pi e^{2}}{A \varepsilon_{0}\left(q+q_{s}\right)} \\
& \times \frac{F_{n, n^{\prime}}(q)}{F_{1,1}(q)\left\{1-\Theta\left(q-2 k_{F}\right) \sqrt{1-\left(2 k_{F} / q\right)^{2}}\right\}}
\end{aligned}
$$

We can simplify things further. Except for very low carrier concentrations, $q<2 k_{F}$ and the heavyside function in the denominator vanishes. In addition, for the first few electron subbands, we have

$$
\frac{F_{n, n^{\prime}}(q)}{F_{1,1}(q)} \approx 1
$$

to within about $5 \%$ for the small values of $q$ of interest and so we arrive at the simple expression

$$
V_{\mathbf{k}, \mathbf{k}^{\prime}}^{n, n^{\prime}}(\mathbf{q})=\frac{2 \pi e^{2}}{A \varepsilon_{0}\left(q+q_{s}\right)}=V(q) .
$$

This is just the strict two-dimensional limit of the screened Coulomb potential and depends only on $|\mathbf{q}|$. The expected reduction in the bare Coulomb potential due to the finite size of the quantum well is very nearly canceled by a corresponding reduction in the RPA dielectric function and so we are justified in using the strict two-dimensional limit for the screened Coulomb potential.

Taking the Pauli exclusion principle into account, the collision integral for electron-electron scattering within the same band, $n$, is given by

$$
\begin{aligned}
{\left[\frac{\partial f_{n}(\mathbf{k}, t)}{\partial t}\right]_{\mathrm{cc}}=} & \sum_{\mathbf{k}^{\prime}}\left\{f_{n}\left(\mathbf{k}^{\prime}, t\right) S_{\mathbf{k}^{\prime}, \mathbf{k}}^{n}\left(1-f_{n}(\mathbf{k}, t)\right)\right. \\
& \left.-f_{n}(\mathbf{k}, t) S_{\mathbf{k}, \mathbf{k}^{\prime}}^{n}\left(1-f_{n}\left(\mathbf{k}^{\prime}, t\right)\right)\right\}
\end{aligned}
$$

where $S_{\mathbf{k}, \mathbf{k}^{\prime}}^{n}$, the rate at which electrons in band $n$ scatter from $\mathbf{k}$ to $\mathbf{k}^{\prime}$, is obtained from the two-body scattering rate by summing over the states of the target electron. Thus

$$
S_{\mathbf{k}, \mathbf{k}^{\prime}}^{n}=\sum_{n^{\prime \prime}, \mathbf{k}^{\prime \prime}} f_{n^{\prime \prime}}\left(\mathbf{k}^{\prime \prime}\right) W_{\mathbf{k}, \mathbf{k}^{\prime \prime}}^{n, n^{\prime \prime}}\left(\left|\mathbf{k}-\mathbf{k}^{\prime}\right|\right)\left[1-f_{n^{\prime \prime}}\left(\mathbf{k}^{\prime \prime}-\mathbf{k}^{\prime}+\mathbf{k}\right)\right]
$$

In the limit of a weak pump, the distribution functions will only be slightly perturbed from thermal equilibrium. Thus

$$
f_{n}(\mathbf{k}, t)=f_{n}^{0}(\mathbf{k})+g_{n}(\mathbf{k}, t),
$$

where the differential distribution function $g_{n}(\mathbf{k}, t)$ is small. The "relaxation-time approximation"' that we make consists of replacing $f_{n}\left(\mathbf{k}^{\prime}, t\right)$ by $f_{n}^{0}\left(\mathbf{k}^{\prime}\right)$ when it is integrated over in Eq. (30). For $f_{n}(\mathbf{k}, t)$ outside the integrals in (30), we use Eq. (32). Thus, for the first term on the right-hand side of (30), $f_{n}\left(\mathbf{k}^{\prime}, t\right)$ is replaced by $f_{n}^{0}\left(\mathbf{k}^{\prime}\right)$ and $1-f_{n}(\mathbf{k}, t)$ is replaced by $1-f_{n}^{0}(\mathbf{k})-g_{n}(\mathbf{k}, t)$. For the term $S_{\mathbf{k}, \mathbf{k}^{\prime}}^{n}$, only the equilibrium distribution functions are used. A similar procedure is used for the second term on the right-hand side of (30). This procedure is different from the standard "linearization" procedure where deviations in $f_{n}(\mathbf{k}, t)$ from equilibrium are considered even when integrated over. Our procedure simplifies the form of the collision integral and is based on the belief that integrals of the change in distribution function should be small. As a consequence of the neglect of these extra terms, one must choose $\mu^{*}$ and $T^{*}$ in the relaxation function $f_{n}^{0}\left(\mathbf{k}, \mu^{*}, T^{*}\right)$ so as to ensure conservation of energy and particle number in the carrier-carrier scattering process.

Making the above relaxation approximation, we obtain

$$
\left[\frac{\partial f_{n}(\mathbf{k}, t)}{\partial t}\right]_{\mathrm{cc}}=-\frac{g_{n}(\mathbf{k}, t)}{\tau_{n}(\mathbf{k})}=-\frac{f_{n}(\mathbf{k}, t)-f_{n}^{0}(\mathbf{k})}{\tau_{n}(\mathbf{k})}
$$

where the relaxation rate is given by 


$$
\begin{aligned}
\frac{1}{\tau_{n}(\mathbf{k})}= & \frac{2 \pi}{\hbar} \sum_{n^{\prime \prime}, \mathbf{k}^{\prime \prime}, \mathbf{q}}|V(q)|^{2}\left\{f_{n}^{0}(\mathbf{k}+\mathbf{q}) f_{n^{\prime \prime}}^{0}\left(\mathbf{k}^{\prime \prime}-\mathbf{q}\right)\left[1-f_{n}^{0}\left(\mathbf{k}^{\prime \prime}\right)\right]+f_{n^{\prime \prime}}^{0}\left(\mathbf{k}^{\prime \prime}\right)\left[1-f_{n}^{0}(\mathbf{k}+\mathbf{q})\right]\left[1-f_{n^{\prime \prime}}^{0}\left(\mathbf{k}^{\prime \prime}-\mathbf{q}\right)\right]\right\} \delta\left(E_{n}(\mathbf{k}+\mathbf{q})\right. \\
& \left.+E_{n^{\prime \prime}}\left(\mathbf{k}^{\prime \prime}-\mathbf{q}\right)-E_{n}(\mathbf{k})-E_{n^{\prime \prime}}\left(\mathbf{k}^{\prime \prime}\right)\right),
\end{aligned}
$$

where $f_{n}^{0}(\mathbf{k})$ is the initial equilibrium distribution function. In our axial approximation, the distribution functions and subband energies depend only on the magnitudes of their arguments. If we take the spherical average of the relaxation operator, we find

$$
\left[\frac{\partial f_{n}(k, t)}{\partial t}\right]_{\mathrm{cc}}=-\frac{f_{n}(k, t)-f_{n}^{0}(k, t)}{\tau_{n}(k)},
$$

where

$$
\frac{1}{\tau_{n}(k)}=\frac{1}{2 \pi} \int_{0}^{2 \pi} d \theta \frac{1}{\tau_{n}(k, \theta)} .
$$

For each subband, we evaluate and store the relaxation rates at $k_{m}$.

\section{Relaxation rates for holes}

The calculation of relaxation rates is more involved in the case of the holes. In the first place, we can no longer assume that the incident and target particles remain in their original subbands. The reason for this will become apparent shortly. We let $W_{n^{\prime}, m^{\prime}}^{n, m}\left(\mathbf{k}, \mathbf{k}^{\prime}, \mathbf{q}\right)$ be the two-body scattering rate at which a hole initially in state $|n, \mathbf{k}\rangle$ scatters off a second hole initially in state $\left|n^{\prime}, \mathbf{k}^{\prime}\right\rangle$ and ends up in a final state $|m, \mathbf{k}+\mathbf{q}\rangle$ having scattered the second hole to the state $\left|m^{\prime}, \mathbf{k}^{\prime}-\mathbf{q}\right\rangle$.

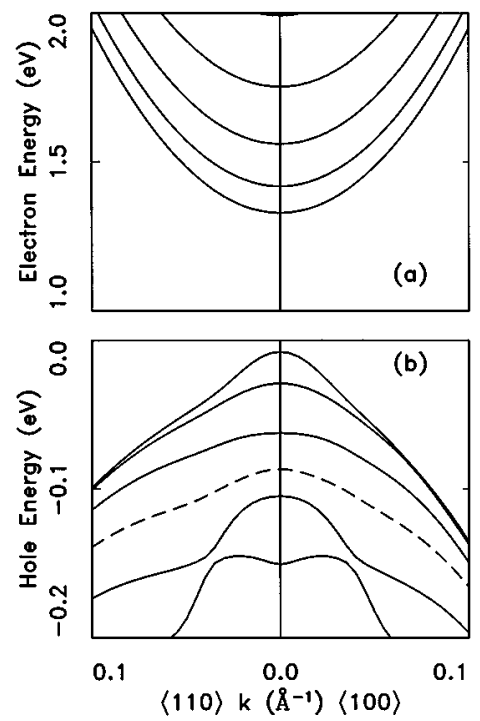

FIG. 3. Computed electronic subband structure along $\langle 110\rangle$ and $\langle 100\rangle$ in the active region of the device. The conduction subbands are shown in (a) and the valence subbands are shown in (b). The heavy-hole subbands are solid lines and the first light-hole level is shown as a dashed line. We include the $100-\AA \mathrm{Ga}_{x} \mathrm{In}_{1-x}$ As SQW and the $25-\AA \mathrm{GaAs}$ bounding layers and assume complete confinement of electrons and holes within the active layer.
For holes the quantum well wave functions are given by

$$
\psi_{n, \mathbf{k}}(\mathbf{r})=\frac{1}{\sqrt{A}} e^{i \mathbf{k} \cdot \mathbf{r}} \sum_{\nu=-3 / 2}^{\nu=3 / 2} F_{n, \mathbf{k}}^{\nu}(z)\left|J=\frac{3}{2}, \nu\right\rangle,
$$

where $\left|J=\frac{3}{2}, \nu\right\rangle$ is the $p$-like spin $\frac{3}{2}$ Bloch state and $F_{n, \mathbf{k}}^{\nu}(z)$ are the envelope functions. Unlike the electron envelope functions, the hole envelope functions depend explicitly on the spin index, $\nu$, as well as the wave vector, $k$. With the more complicated wave functions, the matrix element for the screened Coulomb interaction becomes

$$
V_{n^{\prime}, m^{\prime}}^{n, m}\left(\mathbf{k}, \mathbf{k}^{\prime}, \mathbf{q}\right)=\frac{2 \pi e^{2}}{A \varepsilon_{0} q \varepsilon(q)} F_{n^{\prime}, m^{\prime}}^{n, m}\left(\mathbf{k}, \mathbf{k}^{\prime}, \mathbf{q}\right)
$$

In calculating the dielectric function, $\epsilon(q)$, for Eq. (38), we include only the electrons in the screening. In a static screening approximation, both the electrons and the holes would contribute to the screening. However, the holes are much more massive than the electrons and therefore a dynamic screening model is more appropriate, since the heavier holes are not effective in screening the electrons. To allow for this, sometimes a pseudodynamic screening model is used. In pseudodynamic screening, one neglects all carriers that are heaver than a given carrier in determining the screening. For instance, heavy holes do not screen the light holes, but the light holes do screen the heavy holes. This method is difficult to implement in the strained-layer quantum wells since the valence subbands are not characterized by a single effective mass (cf. Fig. 3) and whether a hole is light or heavy depends upon where it is in a given subband. Thus one cannot easily implement pseudodynamic screening and therefore must use a fully dynamical screening model including the full band structure. This is beyond the scope of the present calculations. As a compromise, we therefore choose to neglect all the holes in the screening. This should be more realistic than a static screening model but will slightly underestimate the effects of screening on the hole scattering. We do not anticipate that these effects should be major since the holes, by virtue of their larger effective masses, are generated by the optical pulse much closer to equilibrium than the electrons. In this simplified screening model, we have

$$
V_{n^{\prime}, m^{\prime}}^{n, m}\left(\mathbf{k}, \mathbf{k}^{\prime}, \mathbf{q}\right)=\frac{2 \pi e^{2}}{A \varepsilon_{0}\left(q+q_{s}\right)} \frac{F_{n^{\prime}, m^{\prime}}^{n, m}\left(\mathbf{k}, \mathbf{k}^{\prime}, \mathbf{q}\right)}{F_{1,1}(q)} .
$$

The form factor is more complicated than for the electrons, however. For holes we have 


$$
\begin{aligned}
F_{n^{\prime}, m^{\prime}}^{n, m}\left(\mathbf{k}, \mathbf{k}^{\prime}, \mathbf{q}\right)= & \int d z \int d z^{\prime} e^{-q\left|z-z^{\prime}\right|} \sum_{\nu} F_{n, \mathbf{k}}^{\nu *}(z) F_{m, \mathbf{k}+\mathbf{q}}^{\nu}(z) \\
& \times \sum_{\nu^{\prime}} F_{n^{\prime}, \mathbf{k}^{\prime}}^{\nu^{\prime} *}\left(z^{\prime}\right) F_{m^{\prime}, \mathbf{k}^{\prime}-\mathbf{q}^{\nu^{\prime}}}^{\nu^{\prime}}\left(z^{\prime}\right) .
\end{aligned}
$$

In the case of electrons, the form factors correct for the finite size of the well and we saw that in the limit of an infinitely thin quantum well, the form factors for electron subbands approached unity. This does not hold in the hole case since the hole envelope functions depend explicitly on the wave vectors of the initial and final states. If we were to ignore the interactions between heavy- and light-hole states, we would obtain two sets of parabolic hole bands. In this case, it would be permissible to make the diagonal approximation and assume that incident and target particles remained in their original subbands. This is because the form factor for a twobody collision in which a hole scatters to a different subband would be small in this case. When interactions between heavy and light holes are included, however, the wave functions become admixtures of heavy- and light-hole states and the form factors for scattering between heavy- and light-hole subbands can no longer be ignored. The diagonal approximation that holes remain in their original subbands is no longer appropriate and we must explicitly allow for hole scattering between different subbands.

The relaxation rate for holes is given by

$$
\begin{aligned}
\frac{1}{\tau_{n}(\mathbf{k})}= & \frac{2 \pi}{\hbar} \sum_{n^{\prime}, m, m^{\prime}, \mathbf{k}^{\prime}, \mathbf{q}}\left|V_{m, m^{\prime}}^{n, n^{\prime}}\left(\mathbf{k}, \mathbf{k}^{\prime}, \mathbf{q}\right)\right|^{2}\left\{f_{m}^{0}(\mathbf{k}+\mathbf{q}) f_{m^{\prime}}^{0}\left(\mathbf{k}^{\prime}-\mathbf{q}\right)\left[1-f_{n^{\prime}}^{0}\left(\mathbf{k}^{\prime}\right)\right]+f_{n^{\prime}}^{0}\left(\mathbf{k}^{\prime}\right)\left[1-f_{m}^{0}(\mathbf{k}+\mathbf{q})\right]\right. \\
& \left.\times\left[1-f_{m^{\prime}}^{0}\left(\mathbf{k}^{\prime}-\mathbf{q}\right)\right]\right\} \delta\left(E_{m}(\mathbf{k}+\mathbf{q})+E_{m^{\prime}}\left(\mathbf{k}^{\prime}-\mathbf{q}\right)-E_{n}(\mathbf{k})-E_{n^{\prime}}\left(\mathbf{k}^{\prime}\right)\right)
\end{aligned}
$$

and we take the angular average of this $k$-dependent relaxation rate to obtain the relaxation rates at $k_{m}$ in the onedimensional model.

Electron-hole scattering is neglected in this calculation. Since the holes are much more massive than the electrons, electron-hole scattering is similar to electron-impurity scattering. That is, the scattering is quasielastic and the main effect is to cause the electrons to relax to their angular averaged distribution. Since the distributions are already angular averaged, the effects of electron-hole scattering should be minimal.

\section{E. Time-dependent optical gain and differential transmission}

The time-dependent optical gain for a given polarization can be expressed in terms of the optical matrix elements, electronic band structure, and time-dependent axial distribution functions as

$$
\begin{aligned}
g^{\lambda}(\hbar \omega, t) \propto & \sum_{n_{c}, n_{v}} \sum_{m}\left|\boldsymbol{\lambda} \cdot \mathbf{P}_{n_{c}, n_{v}}\left(k_{m}\right)\right|^{2} \\
& \times\left[f_{n_{c}}\left(k_{m}, t\right)+f_{n_{v}}\left(k_{m}, t\right)-1\right] 2 \pi \\
& \times \int_{k_{m}-\Delta k / 2}^{k_{m}+\Delta k / 2} d k k \delta\left(E_{n_{c}}(k)-E_{n_{v}}(k)-\hbar \omega\right) .
\end{aligned}
$$

It should be noted that in the absence of carriers, the optical gain is just the negative of the absorption coefficient. In calculating (42), the energies of the bands are shifted by 23 $\mathrm{meV}$ to match the disagreement between the theoretical band edge calculated with the Luttinger model and the experimental edge. This discrepancy is most likely due to band-gap renormalization resulting from the injected background carriers.
Spectral broadening of the probe pulse is taken into account by replacing the Dirac $\delta$ function in the gain equation with a Lorentzian function:

$$
\delta(x) \rightarrow \frac{1}{\pi} \frac{\hbar / \tau_{\text {probe }}}{x^{2}+\left(\hbar / \tau_{\text {probe }}\right)^{2}}
$$

where

$$
\hbar / \tau_{\text {probe }}=\frac{\hbar}{2}\left(1 / \tau_{e}+1 / \tau_{h}\right)
$$

is the associated energy broadening factor and $1 / \tau_{e}, 1 / \tau_{h}$ are the electron and hole scattering rates. In calculating the broadening factor, we must not only take into account carrier-phonon scattering, but also carrier-carrier scattering. We take $\hbar / \tau_{\text {probe }}=22.5 \mathrm{meV}$ based on the calculation of Asada. $^{21}$ In addition, the time-dependent optical gain $g^{\lambda}(\hbar \omega, t)$ is convoluted in time with the probe pulse.

The time-dependent transmission coefficient is defined as the ratio of transmitted to incident power. ${ }^{22}$ In the thin sample limit, where $L$ is the thickness of the sample, we have

$$
\frac{\Delta T^{\lambda}}{T^{\lambda}} \approx\left[g^{\lambda}(\hbar \omega, t)-g^{\lambda}(\hbar \omega, t \rightarrow-\infty)\right] L .
$$

In practice, we theoretically calculate only the change in absorption and do not multiply by a given length scale since the experimental sample lengths vary from sample to sample. The units in our plots are therefore arbitrary. (In addition, the plots have also been scaled so that the numbers on the graphs are easier to read.) In the experiments, the typical values for $\Delta T / T$ are $0.1-2 \%$. This depends, however, on the intensity of the pump pulse since in the low-intensity limit, the magnitude of the signal depends on the number of carriers created. 

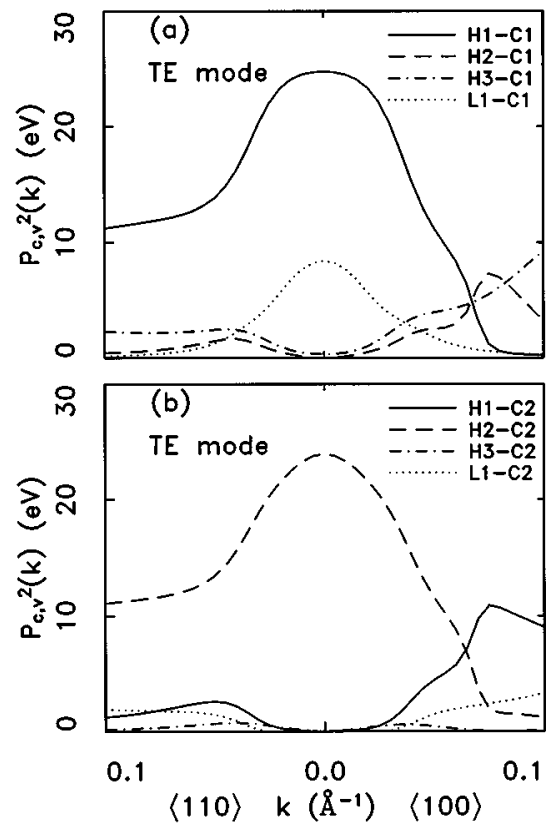

FIG. 4. Squared optical matrix elements along $\langle 110\rangle$ and $\langle 100\rangle$ for dipole-allowed TE transitions between the first four valence subbands and the first (a) and second (b) conduction subbands.

\section{THEORETICAL RESULTS}

\section{A. Band structure}

The active region of the device consists of the $100-\AA$ $\mathrm{Ga}_{0.87} \mathrm{In}_{0.13}$ As SQW and the two $25-\AA$ GaAs bounding layers. We assume that the carriers are completely confined within the $\mathrm{Al}_{x} \mathrm{Ga}_{1-x} \mathrm{As}$ GRIN structure and take the barriers to be infinite. The SQW barriers are taken to be finite and we allow the carriers to tunnel into the GaAs bounding layers. The Brillouin zone lies in the $x-y$ plane and the computed subband structures near $k=0$ are shown in Fig. 3 along the $\langle 110\rangle$ and $\langle 100\rangle$ directions.

The conduction subbands in Fig. 3(a) are isotropic and parabolic and the electron states are derived from $s$-like Bloch states. By convention, we label the electron subbands $C_{n}$ where $n=1,2,3, \ldots$ denotes the subband index. The hole subbands in Fig. 3(b) are nonparabolic and anisotropic and in general the hole states are mixtures of heavy- and light-hole Bloch functions. By convention, we label the hole subbands $H_{n}$ or $L_{n}$ depending on their heavy- and light-hole character at the zone center. The terms "heavy" and "light" refer to the effective masses along the $z$ direction. Heavy holes are heavy along the $z$ direction but light in the $x-y$ plane, while light holes are light in the $z$ direction but heavy in the $x-y$ plane. In the figure, the heavy-hole subbands are shown as solid lines and the first light-hole subband is shown as a dashed line.

\section{B. Optical matrix elements}

The squared optical matrix elements for dipole-allowed transitions between valence and conduction subbands are shown in Fig. 4 for TE polarization. We plot the squared optical matrix elements as a function of wave vector in the $\langle 110\rangle$ and $\langle 100\rangle$ directions for transitions involving the first four valence and first two conduction subbands. The squared

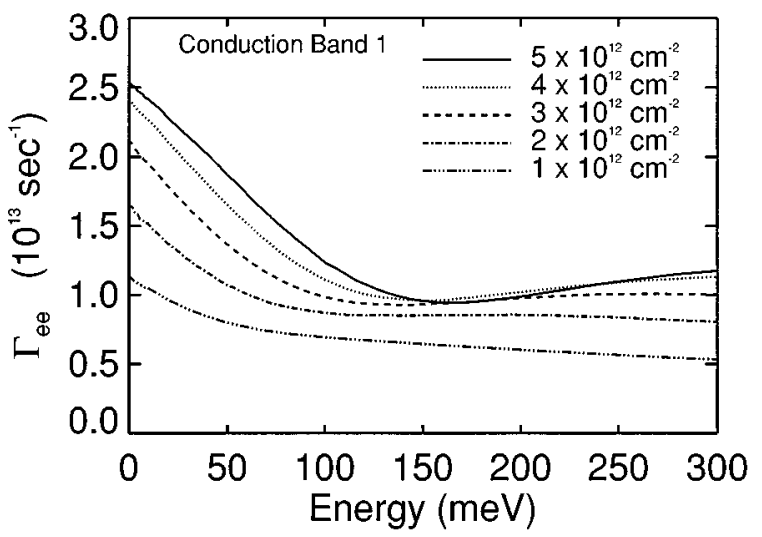

FIG. 5. Energy-dependent carrier-carrier relaxation rates at 300 $\mathrm{K}$ for electrons in the first conduction subband. The carrier-carrier relaxation rate depends on the lattice temperature and injected carrier density. As the carrier density is increased, carrier-carrier scattering becomes more effective in thermalizing the electron distribution function.

optical matrix elements are seen to be anisotropic and to strongly depend on wave vector.

At the zone center, the squared optical matrix element is proportional to the product of the squared momentum matrix element between the electron and hole envelope functions. The hole envelope function at $k=0$ will vanish unless the subband indices for the valence and conduction subbands are the same. This is the $\Delta n=0$ selection rule for optical transitions between quantum well subbands. This is evident in Fig. 4 , where the strongest optical matrix elements at the zone center correspond to $H_{1}-C_{1}, L_{1}-C_{1}$, and $H_{2}-C_{2}$ transitions.

Our model includes the effects of mixing of light- and heavy-hole states in the Luttinger Hamiltonian. As we move away from the zone center, we find that the oscillator strength is redistributed among the various subbands. As can be seen in Fig. 4, the forbidden transitions pick up oscillator strength at the expense of the allowed transitions.

\section{Carrier-carrier relaxation rates}

For the SQW laser device shown in Fig. 2, the polar optical phonon scattering-out rates for electrons and holes were reported in our earlier paper. ${ }^{15}$ In the present paper we consider both polar optical phonon scattering and carrier-carrier scattering in the effective relaxation model. In general, the relaxation rates, $1 / \tau_{n}$, for carrier-carrier scattering in subband $n$ depend on the lattice temperature, the initial carrier concentration, and the carrier energy (or equivalently, the carrier wave vector). The energy-dependent relaxation rate at a lattice temperature of $300 \mathrm{~K}$ for electrons in the first conduction subband is shown in Fig. 5 for several initial carrier concentrations. The relaxation rate for electron-electron scattering is seen to increase as the background carrier concentration is raised. We can anticipate that at low background carrier concentrations, carrier-carrier scattering can be neglected in comparison with polar optical phonon scattering while at high background concentrations, carrier-carrier scattering will be the dominant scattering mechanism. Estimates indicate that for carrier concentrations above $1 \times 10^{12} \mathrm{~cm}^{-2}$, carrier-carrier scattering is important and must be included. 


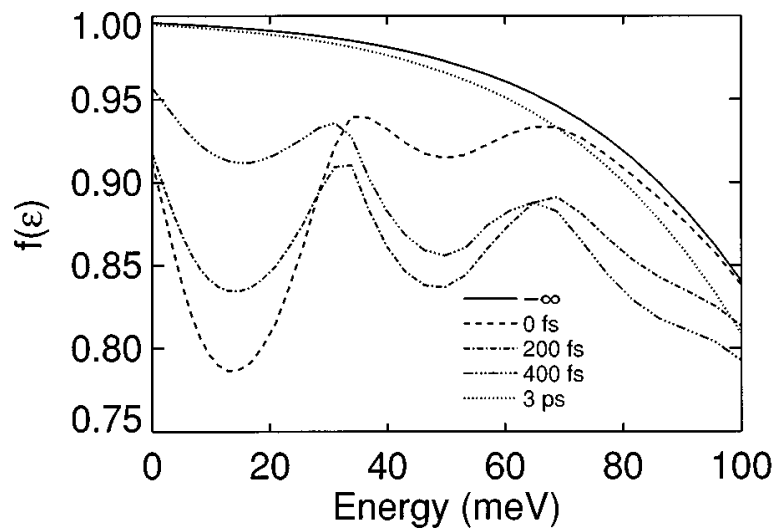

FIG. 6. Time-dependent electron distribution function in the first conduction subband in the presence of a pump pulse. The pump is in the gain regime and electrons are depleted through stimulated emission and subsequently relax through polar optical phonon scattering.

\section{Time-dependent distribution functions}

To illustrate the effects of carrier-carrier scattering on electron dynamics at moderately high injected carrier densities, we set the lattice temperature to $300 \mathrm{~K}$ and the injected carrier concentration to $5.0 \times 10^{12} \mathrm{~cm}^{-2}$ and compute the time-dependent distribution functions in the presence of a strong pump pulse with a pulse energy of $E_{0}=0.4 \mathrm{fJ}$ (as defined in Ref. 15), a central energy of $\hbar \omega=1.358 \mathrm{eV}$, a spectral width of $\sigma=30 \mathrm{meV}$, and a temporal width of $\tau_{0}=200$ fs. For this combination of lattice temperature, injected carrier concentration, and central pulse energy, the pump is in the gain regime and will act to deplete carriers from the active region of the device through stimulated emission. In simulating the carrier dynamics of the device, the distribution functions were first computed with polar optical phonon scattering as the only scattering mechanism. The results for the first electron subband are shown in Figs. 6 and 7 with only polar optical phonon scattering.

In Fig. 6, we plot the electron distribution as a function of energy relative to the bottom of the first conduction subband for several different times. The different curves are: before the pulse (solid line), center of the pulse (dashed line), $200 \mathrm{fs}$ (dash-dotted line), 400 fs (dash-multi dotted line) and 3 ps (dotted line). At $t=-\infty$, the initial electron distribution is a

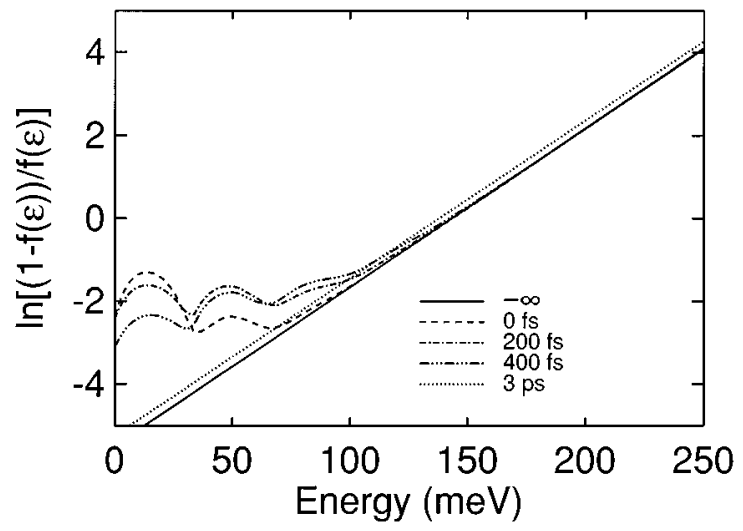

FIG. 7. $\ln \{[1-f(E)] / f(E)\}$ plotted as a function of energy for the time-dependent electron distribution functions shown in Fig. 6.
Fermi function determined by the subband structure of the device, the lattice temperature, and the injected carrier concentration. At $t=0$, the pump reaches its maximum intensity and a hole is burned in the electron distribution function near $18 \mathrm{meV}$. As time progresses, electrons at higher energy are scattered into this hole by emission of polar optical phonons and a series of dips appear in the electron distribution function spaced a LO phonon energy apart. After a few picoseconds, a quasiequilibrium Fermi distribution is established.

It is useful to plot the quantity

$$
\ln \left(\frac{1-f(E)}{f(E)}\right)
$$

as a function of energy. If $f(E)$ is a Fermi distribution, we obtain a straight line,

$$
\ln \left(\frac{1-f(E)}{f(E)}\right)=\beta E-\beta \mu,
$$

where the intercept $\beta \mu$ is proportional to the chemical potential $\mu$ and the slope $\beta=1 / k_{B} T$ is the reciprocal of the temperature in units of energy. In Fig. 7 we plot

$$
\ln \left(\frac{1-f(E)}{f(E)}\right)
$$

for the distribution functions depicted in Fig. 6. At the beginning and end of the simulation, the distribution function is seen to be a Fermi function with a temperature of $300 \mathrm{~K}$ (the initial lattice temperature). Note that the chemical potential shifts (as seen by the change in intercept) due to the fact that the pump laser has removed carriers from the device. Around $t=0$, the distribution function is nonthermal and the hole burning peak and associated phonon replicas are clearly visible in the energy range below $100 \mathrm{meV}$. In the high-energy tail of the distribution above $100 \mathrm{meV}$, the distribution functions are well characterized by Fermi functions at the initial lattice temperature, but with a slowly varying chemical potential. The effective "electron temperature" does not change during the relaxation.

We next computed the distribution functions including both polar optical phonon and carrier-carrier scattering in the simulation. The results for the first electron subband are shown in Fig. 8 where the electron distribution is plotted as a function of energy. The pump pulse and the initial electron distribution are the same as in Fig. 6. As the pump reaches its maximum intensity at $t=0$, a hole is again burned in the electron distribution function near $18 \mathrm{meV}$. The laser acts to deplete carriers and drive the device to transparency while the scattering mechanisms act to oppose this tendency and restore an equilibrium distribution. Comparing Figs. 6 and 8 we see that the hole burning peak is shallower in the presence of carrier-carrier scattering as one would expect. Carrier-carrier scattering also acts to smooth out the phonon replicas at later times. We find that more carriers are depleted from the sample when carrier-carrier scattering is included.

In Fig. 9 we plot

$$
\ln \left(\frac{1-f(E)}{f(E)}\right)
$$




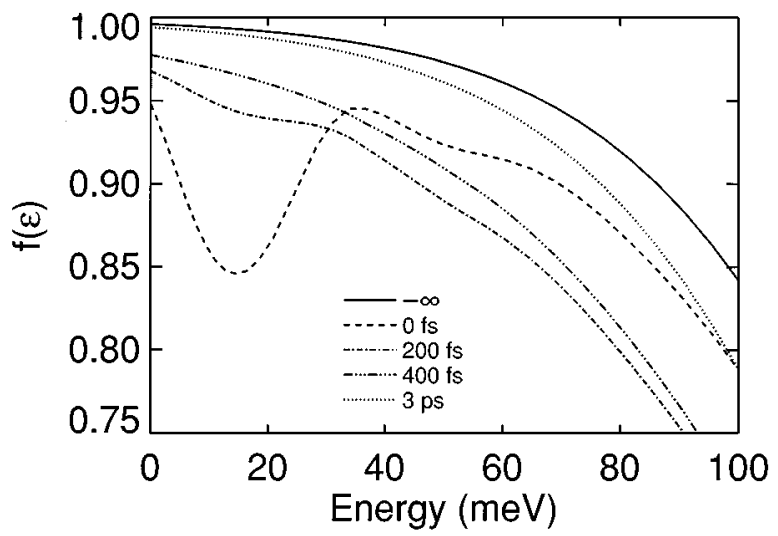

FIG. 8. Time-dependent electron distribution function in the first conduction subband in the presence of a pump pulse. The pump is in the gain regime and electrons are depleted through stimulated emission and subsequently relax through both polar optical phonon scattering and carrier-carrier scattering.

as a function of energy for the first conduction subband for the distribution functions depicted in Fig. 8 including both carrier-carrier and polar optical phonon scattering. When the pump is at maximum intensity, the distribution function is nonthermal around the pump energy. Carrier-carrier scattering quickly thermalizes the distribution functions and heats the high-energy tails of the distribution functions (0-400 fs). Eventually ( $3 \mathrm{ps})$, the temperature of the tail relaxes back to room temperature. Neglecting the hole burning peak near the center of the pulse, the distribution functions can be characterized by a Fermi distribution with a time-varying effective temperature and chemical potential.

We plot the time-dependent distribution functions for the first hole subband in Fig. 10 for optical phonon scattering only and in Fig. 11 we show the corresponding results with carrier-carrier scattering included. The time for the various curves are the same as in Figs. 6-9. In Fig. 10, one sees the relaxation process of the hole distribution functions occurring through optical phonon scattering. When carrier-carrier scattering is turned on, the phonon replicas disappear and the carrier-carrier scattering acts to thermalize the distribution.

\section{COMPARISON OF THEORY AND EXPERIMENT}

For the device shown in Fig. 2, time-dependent differential transmission spectra have been measured at different

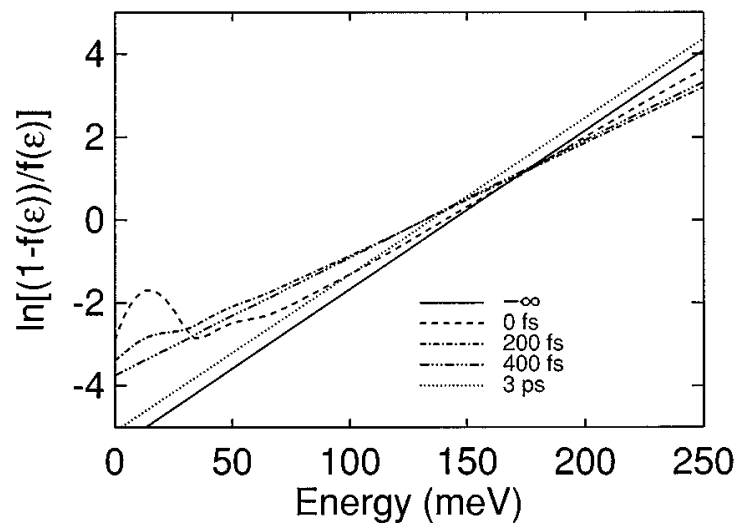

FIG. 9. $\ln \{[1-f(E)] / f(E)\}$ plotted as a function of energy for the time-dependent electron distribution functions shown in Fig. 8.

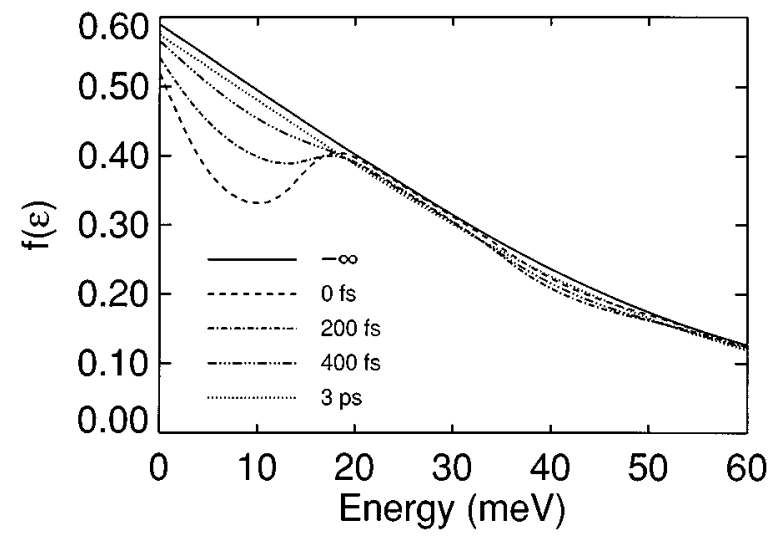

FIG. 10. Time-dependent hole distribution function in the first valence subband in the presence of a pump pulse. The pump pulse is the same as in Fig. 6 and the holes relax by means of polar optical phonon scattering.

probe frequencies. These measurements can be directly compared with our theoretically predicted differential transmission spectra. Depending on the position of the pump relative to the transparency point, the pump can either be in the gain or loss regime, that is, the pump can either create or deplete carriers.

\section{A. Differential transmission spectrum: Pump in the gain regime}

We first consider what happens when the injection current is high enough for the pump to be in the gain regime. We set the injection current to $4.2 \mathrm{~mA}$, which corresponds to an injected carrier concentration of $2.1 \times 10^{12} \mathrm{~cm}^{-2}$. For this injection current, the transparency point is at $925 \mathrm{~nm}$ and the pump is at $935 \mathrm{~nm}$. This places the pump in the gain regime close to the transparency point. The pump is amplified on passing through the device and electrons and holes recombine through stimulated emission. Consequently, the differential transmission is negative.

The experimentally measured differential transmission spectra are shown as a surface plot in Fig. 12 in which the negative of the differential transmission is measured as a

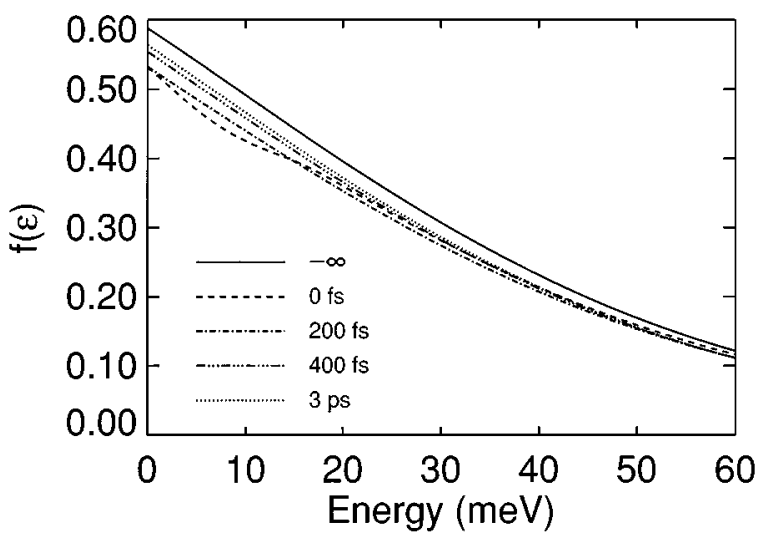

FIG. 11. Time-dependent hole distribution function in the first valence subband in the presence of a pump pulse. The pump pulse is the same as in Fig. 6 and the holes relax by means of both polar optical phonon and carrier-carrier scattering. 


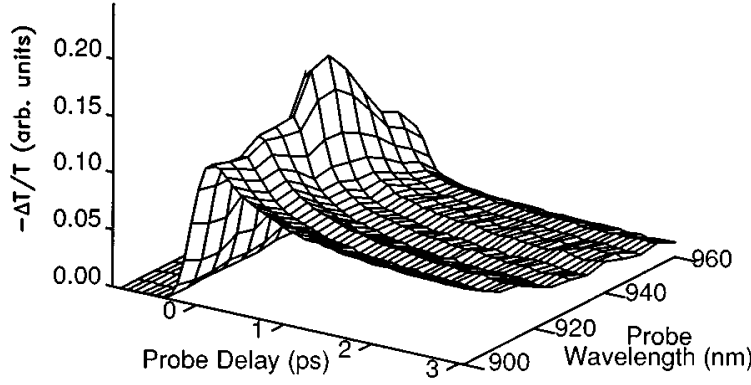

FIG. 12. Experimental data showing femtosecond spectral dependence of transient gain for 4.2-mA bias with the pump in the gain region. The transparency point is at $925 \mathrm{~nm}$ and the pump is at $935 \mathrm{~nm}$. The vertical axis is the negative of the transmission change and the differential transmission is measured as a function of probe wavelength and probe delay.

function of probe delay and probe wavelength. ${ }^{23}$ We can examine the data by taking slices through this surface. In Fig. 13(a), we plot differential transmission as a function of probe wavelength measured at several delay times and in Fig. 13(b) we plot the differential transmission as a function of probe delay at several different probe wavelengths. Around zero time delay, sharp transmission decreases are observed with a spectral peak at the pump wavelength (935 $\mathrm{nm})$ with no spectral shift and a time-resolution-limited recovery. At intermediate time delays, gain changes are mediated by carrier heating and a decrease in the carrier population. ${ }^{23}$ Much stronger carrier heating and carrier population-induced transmission decreases are observed for probe wavelengths close to the transparency point.
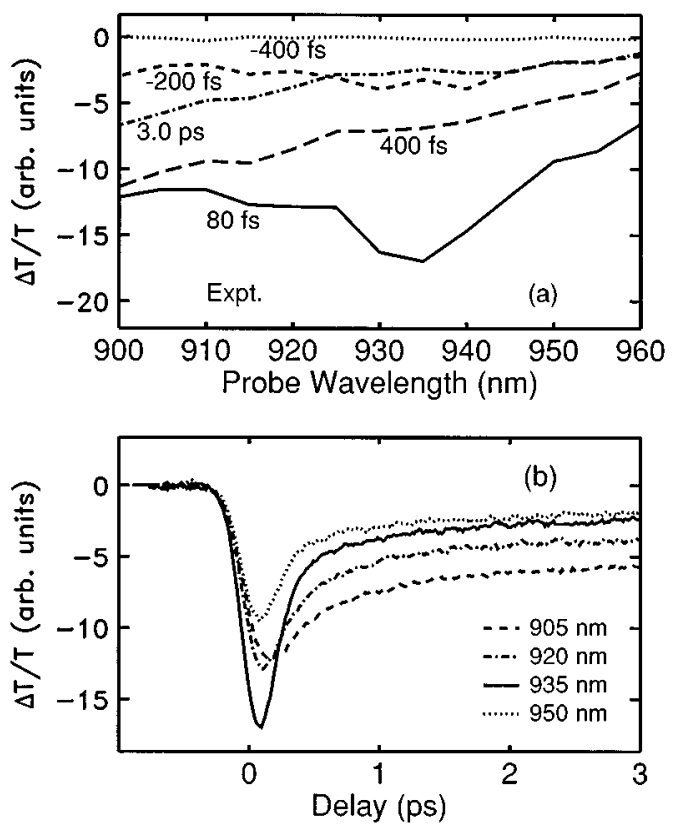

FIG. 13. Selected slices through the experimental differential transmission data seen in Fig. 12. In (a) the differential transmission is plotted as a function of probe wavelength at several different values of the probe delay while in (b) the differential transmission is plotted as a function of probe delay for several values of the probe wavelength.

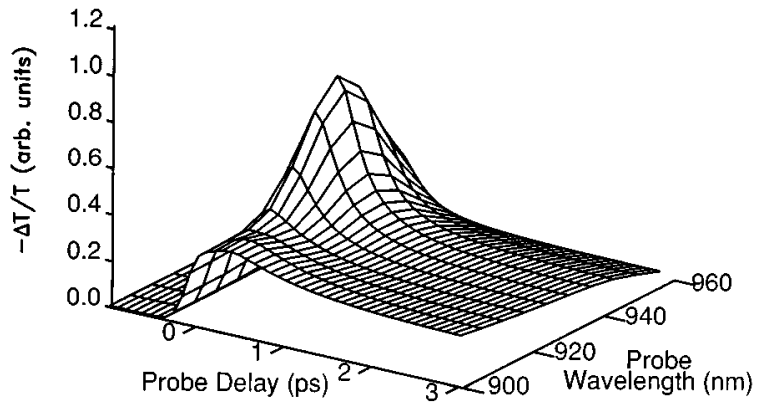

FIG. 14. Theoretically calculated differential transmission spectra for the experimental situation depicted in Fig. 12. Carrier relaxation is by means of polar optical phonon scattering only.

To compare these results with theory and to better appreciate the role of carrier-carrier scattering in the gain dynamics, we compute theoretical differential transmission spectra with and without carrier-carrier scattering. The computed differential transmission spectra as a function of probe delay and probe wavelength in the absence of carrier-carrier scattering is shown in Fig. 14. In Fig. 15(a) we plot the computed differential transmission as a function probe wavelength at several delay times and in Fig. 15(b) the computed differential transmission as a function of probe delay is shown for several different probe wavelengths. In Fig. 14, we can clearly see a large peak in the negative differential transmission centered around the pump wavelength similar to the experimental curve. Electrons and holes are depleted from the lowest-lying conduction and valence subbands and the gain in the vicinity of the pump is suppressed, resulting in a negative differential transmission centered on the pump
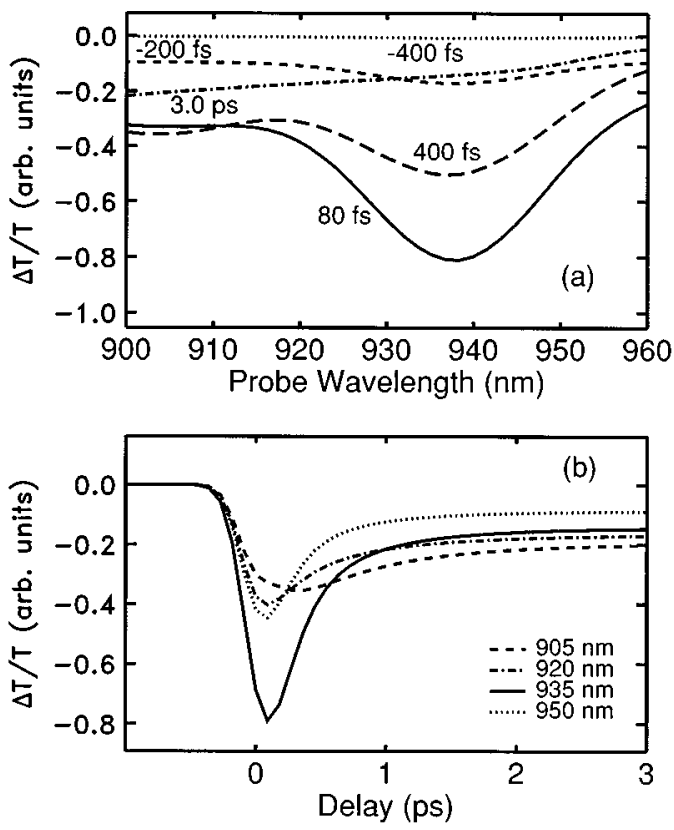

FIG. 15. Selected slices through the theoretical differential transmission spectra seen in Fig. 14. In (a) the differential transmission is plotted as a function of probe wavelength at several different values of the probe delay while in (b) the differential transmission is plotted as a function of probe delay for several values of the probe wavelength. 


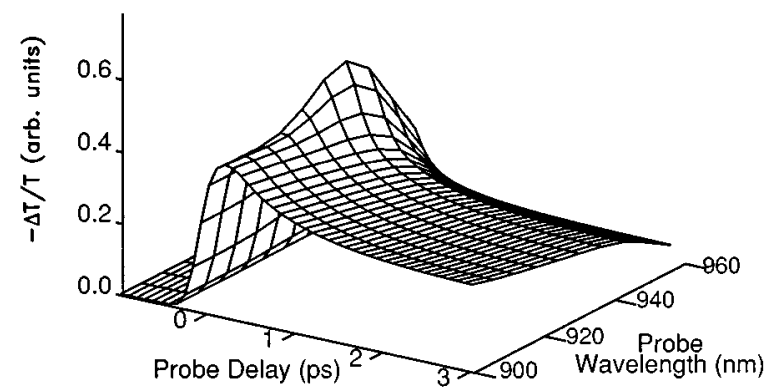

FIG. 16. Theoretically calculated differential transmission spectra for the experimental situation depicted in Fig. 12. Carrier relaxation is by means of both polar optical phonon and carrier-carrier scattering.

wavelength. After the pump dies away, scattering restores a quasiequilibrium gain spectrum on a time scale of a few picoseconds. In Fig. 14 the thermalization of electrons and holes is entirely due to scattering with polar optical phonons. The secondary peak seen near $910 \mathrm{~nm}$ is a phonon replica. Phonon replicas arises when carriers are scattered by an integral number of optical phonon energies and jump into the electron and hole states depleted by the pump.

The computed differential transmission in the presence of carrier-carrier scattering is shown in Fig. 16. In Fig. 17(a) we plot the computed differential transmission as a function probe wavelength at several delay times and in Fig. 17(b) the computed differential transmission as a function of probe delay is shown for several different probe wavelengths. In general, any scattering mechanism will act to restore a quasiequilibrium distribution. The inclusion of carrier-carrier scattering in our model suppresses the dip observed in the
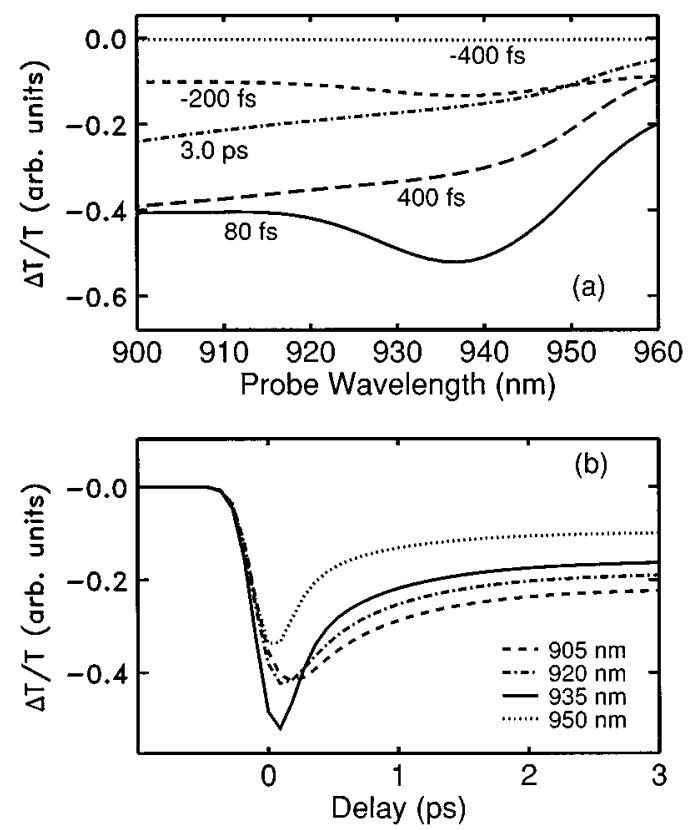

FIG. 17. Selected slices through the theoretical differential transmission spectra seen in Fig. 16. In (a) the differential transmission is plotted as a function of probe wavelength at several different values of the probe delay while in (b) the differential transmission is plotted as a function of probe delay for several values of the probe wavelength.

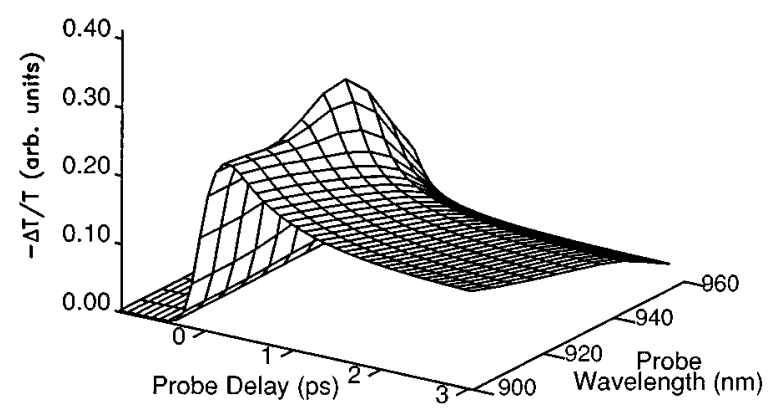

FIG. 18. Theoretically calculated differential transmission spectra for the experimental situation depicted in Fig. 12. Carrier relaxation is by means of both polar optical phonon and carrier-carrier scattering and the probe signal is modified by the suppression of the Coulomb enhancement factor.

differential transmission around the pump frequency and smooths out the phonon replicas. Agreement between these curves and the experiments is better than with phonon scattering only. While inclusion of carrier-carrier scattering smooths out the sharp peaks in the results with only phonon scattering (Figs. 14 and 15), the agreement between experiment and theory with only phonon scattering is not too bad, considering it is much easier to calculate the effects of phonon scattering only.

The Sommerfeld enhancement factor used in calculating the optical matrix elements has not been reduced due to carrier screening. To estimate the importance of screening, we can recalculate the differential transmission without the Sommerfeld factor. This is done in Figs. 18 and 19 where we plot the differential transmission with carrier-carrier and op-
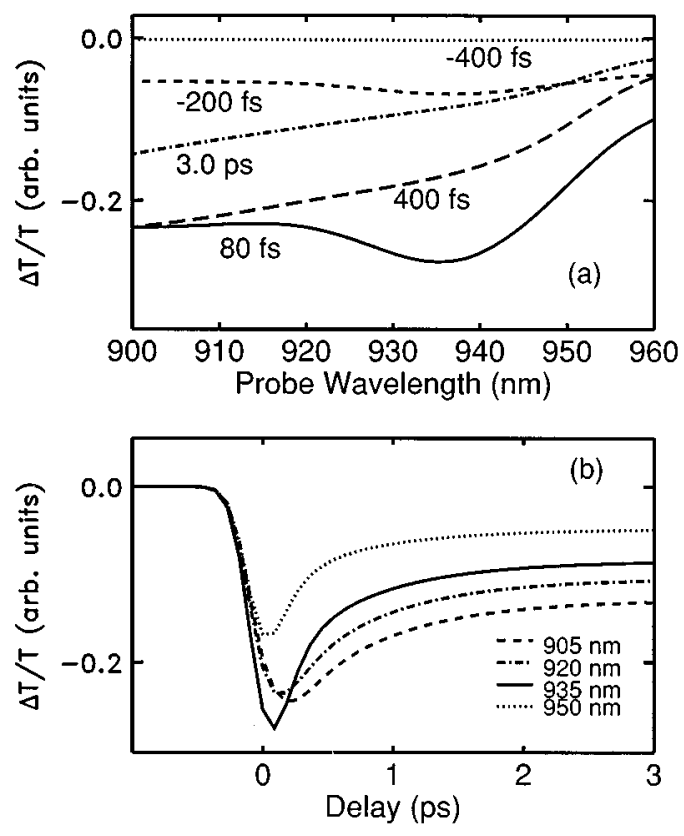

FIG. 19. Selected slices through the theoretical differential transmission spectra seen in Fig. 18. In (a) the differential transmission is plotted as a function of probe wavelength at several different values of the probe delay while in (b) the differential transmission is plotted as a function of probe delay for several values of the probe wavelength. 


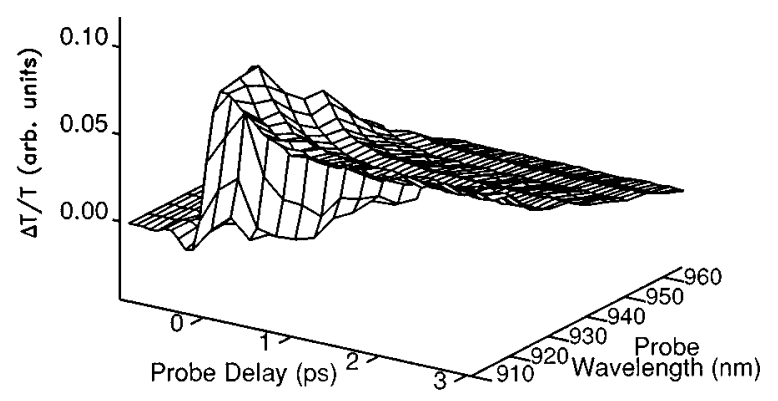

FIG. 20. Experimental data showing femtosecond spectral dependence of transient gain for 3.0-mA bias with the pump in the loss region. The transparency point is at $935 \mathrm{~nm}$ and the pump is at $925 \mathrm{~nm}$. The differential transmission is measured as a function of probe wavelength and probe delay.

tical phonon scattering, but no Sommerfeld factor. In Fig. 19(a) the differential transmission is shown as a function of probe wavelength at several delay times and in Fig. 19(b) the computed differential transmission as a function of probe delay is shown for several different probe wavelengths. While the inclusion of the enhancement factor significantly changes the overall magnitude of the signal, it only slightly modifies the wavelength dependence. Details of the screening effects on the Coulomb enhancement should therefore not be extremely important for the overall shape of the results.

\section{B. Differential transmission spectrum: Pump in the loss regime}

We next consider what happens when the injection current is low enough for the pump to be in the loss regime. When we set the injection current to $3 \mathrm{~mA}$, the injected carrier concentration is $1.41 \times 10^{12} \mathrm{~cm}^{-2}$. The transparency point is now $935 \mathrm{~nm}$ and the pump is at $925 \mathrm{~nm}$. This places the pump in the loss regime. The pump will now be attenuated on passing through the device and it will generate electron-hole pairs through stimulated absorption.

The experimentally measured differential transmission spectra are shown in Fig. 20. Here we plot the experimentally measured (positive) differential transmission changes as a function of probe wavelength and probe delay. The pump is fixed at $925 \mathrm{~nm}$ with a bias current of $3 \mathrm{~mA}$, corresponding to a carrier concentration of $1.4 \times 10^{12} \mathrm{~cm}^{-2}$. The transparency point was at $935 \mathrm{~nm}$ and the probe wavelength was tuned from 910 to $965 \mathrm{~nm}$ in increments of $5 \mathrm{~nm}$. At zero time delay, we observe a sharp transmission decrease. This has been attributed to either two photon absorption or details of the generation mechanism not included in our quasiclassical method. To account for this in our calculations, we add an additional contribution to the differential transmission signal proportional to the convolution of the pump and probe pulse. This initial negative transient is followed by a fast rise, induced by the inverted spectral hole burning and carrier cooling effects, ${ }^{24}$ which reached a maximum at a time delay of $120 \mathrm{fs}$. A spectral peak at $920 \mathrm{~nm}$ is observed, with a $5 \mathrm{~nm}$ blue shift relative to the pump central wavelength. At 400 fs time delay, this spectral peak is smoothed out completely, and the nonlinear gain is induced by carrier cooling and carrier concentration increase. The carrier cooling effects
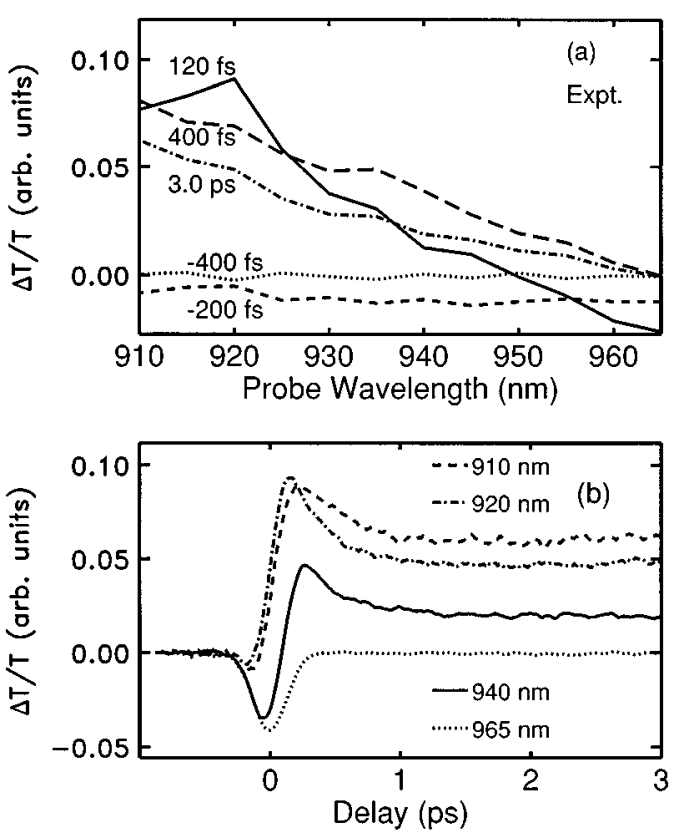

FIG. 21. Selected slices through the experimental differential transmission data seen in Fig. 20. In (a) the differential transmission is plotted as a function of probe wavelength at several different values of the probe delay while in (b) the differential transmission is plotted as a function of probe delay for several values of the probe wavelength.

relaxed through exchange energies with the lattice in $\sim 1 \mathrm{ps}$. After 1 ps a steady spectral response is reached which was induced only by carrier concentration increases and relaxed on a much longer time scale.

Since the pump wavelength is not far from the transparency point, weak inverted spectral hole burning effects are expected. This agrees with experimental observations which show a fast positive transient having a weak spectral peak at $920 \mathrm{~nm}$. The probe transmission changes induced by carrier cooling and carrier concentration increase are comparatively much stronger and dominate the measured response throughout the whole spectrum.

In Fig. 21(a) we plot the experimental differential transmission as a function of probe wavelength measured at several delay times and in Fig. 21(b) we plot the differential transmission as a function of probe delay at several different probe wavelengths.

In Figs. 22 and 23, we plot our computed differential transmission as a function of probe delay and probe wavelength for a simulation in which only polar optical phonon scattering is included. In Figs. 24 and 25, our computed differential transmission includes the effects of both polar optical phonon and carrier-carrier scattering. Again, as can be seen, the inclusion of carrier-carrier scattering improves the agreement between theory and experiment, the net result being that carrier-carrier scattering smooths out sharp structures in the differential transmission. Comparison of Figs. 24 and 20 show that in our model, it appears that the carriercarrier scattering is slightly too strong. This could possibly result from (1) the use of the screening model discussed earlier, (2) the use of a relaxation time approximation, or (3) subtle differences in the experimental and theoretical initial conditions. It warrants future investigations. 


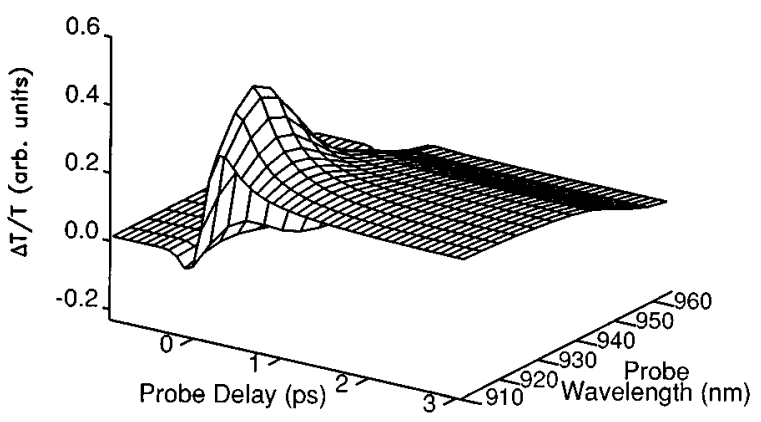

FIG. 22. Theoretically calculated differential transmission spectra for the experimental situation depicted in Fig. 21. Carrier relaxation is by means of polar optical phonon scattering.

\section{Injection current dependence and two-photon absorption}

In addition to computing differential transmission spectra with the pump in the gain and loss regions, we have also studied in detail the dependence of differential transmission on injection current for fixed pump and probe wavelengths. The pump wavelength is fixed at $920 \mathrm{~nm}$ (43 meV above the band gap) and the probe wavelength is fixed at $942 \mathrm{~nm}$ (11 meV above the band gap). In Fig. 26(a), we show the experimentally measured differential transmission spectra at several different injection currents as functions of the probe delay. The instantaneous decrease in probe transmission near zero probe delay seen in all the traces is the result of possible two-photon absorption as discussed earlier. The steplike transmission changes at long time delay are produced by net changes in carrier concentration through interband stimu-
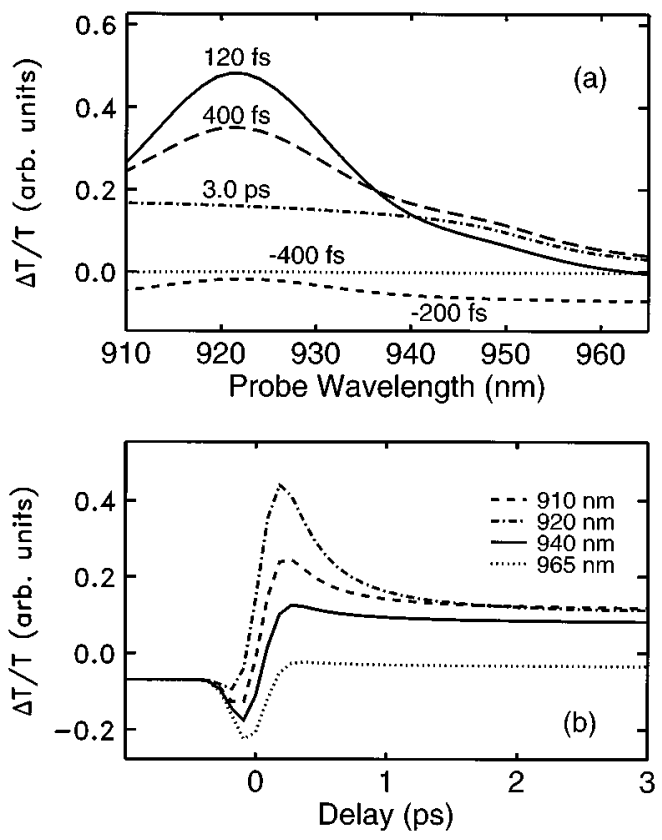

FIG. 23. Selected slices through the theoretical differential transmission spectra seen in Fig. 22. In (a) the differential transmission is plotted as a function of probe wavelength at several different values of the probe delay while in (b) the differential transmission is plotted as a function of probe delay for several values of the probe wavelength.

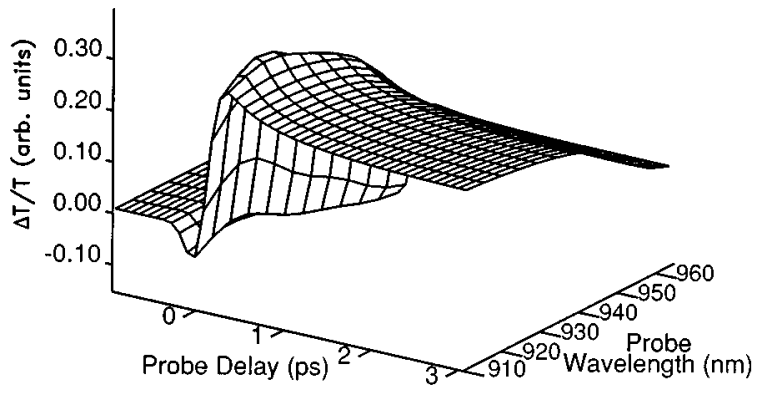

FIG. 24. Theoretically calculated differential transmission spectra for the experimental situation depicted in Fig. 20. Carrier relaxation is by means of both polar optical phonon and carrier-carrier scattering.

lated transitions, and depend on the position of the pump within the gain spectrum. The pump is at the transparency point for an injection current of $4.85 \mathrm{~mA}$. The pump is in the loss regime for injection currents less than $4.85 \mathrm{~mA}$, and in the gain regime for injection currents greater than $4.85 \mathrm{~mA}$. Peak-to-peak transmission changes are around $1 \% .^{24}$

Figure 26(b) shows our computed differential transmission spectra at the probe energy as a function of time delay for several injection carrier concentrations, including the effects of polar optical phonon scattering but ignoring carriercarrier scattering. We see that our calculation correctly reproduces the overall trends seen in the experiments. At low injected carrier concentrations, the pump is in the loss regime and carriers are injected into the quantum well by stimulated absorption. Likewise, at high carrier concentra-
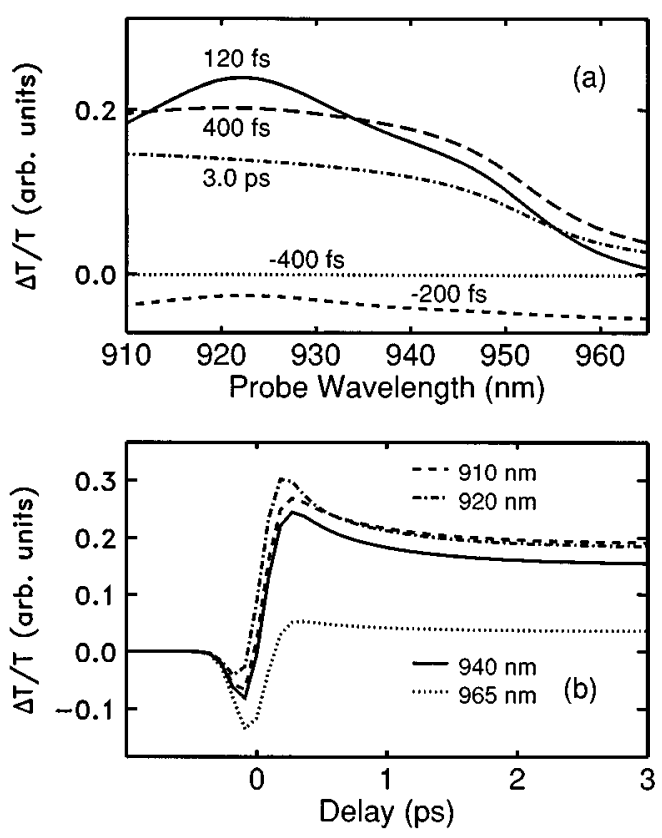

FIG. 25. Selected slices through the theoretical differential transmission spectra seen in Fig. 24. In (a) the differential transmission is plotted as a function of probe wavelength at several different values of the probe delay while in (b) the differential transmission is plotted as a function of probe delay for several values of the probe wavelength. 


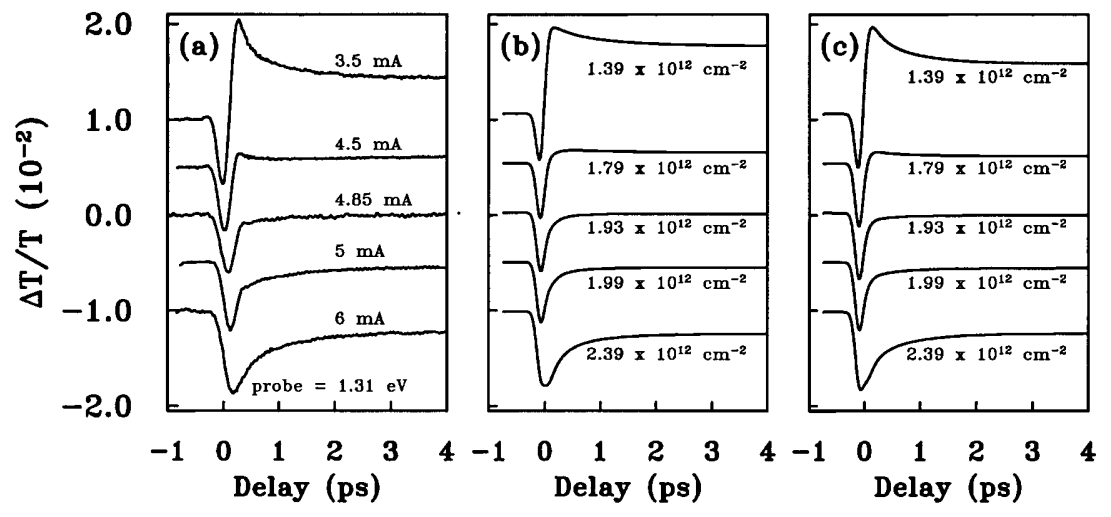

FIG. 26. Differential transmission spectra as a function of probe delay for several injection currents. The pump and probe wavelengths are fixed at 43 and $11 \mathrm{meV}$ above the band gap. In (a) the experimental data are shown. In (b) the computed differential transmission is shown for a simulation with polar-optical-phonon scattering and in (c) the computed differential transmission is shown with both polar optical and carrier-carrier scattering included. tions, the pump is in the gain regime and carriers are extracted from the quantum well through stimulated emission.

Finally, in Fig. 26(c) we plot the computed differential transmission spectra at the probe energy as a function of time delay for several injection currents including the effects of both polar optical phonon and carrier-carrier scattering. Comparing Figs. 26(c) and 26(b) with the experimental curves in Fig. 26(a), we see that the inclusion of carriercarrier scattering improves the overall agreement between theory and experiment. In particular, the slow decrease at long times seen in the top curve of Fig. 26(a) (the 3.5-mA case), is reproduced in Fig. 26(c).

\section{CONCLUSIONS}

We have presented measurements and calculations of the femtosecond gain dynamics in $\mathrm{In}_{1-x} \mathrm{Ga}_{x} \mathrm{As} / \mathrm{Al}_{1-y} \mathrm{Ga}_{y} \mathrm{As}$ strained-layer single-quantum-well diode lasers. A multiplewavelength femtosecond pump-probe technique was used to study transient gain dynamics in an active diode laser under various injection conditions. The pump and probe wavelengths can be varied independently and the differential transmission studied as a function of probe wavelength and probe delay.

To model the experimentally measured differential transmission spectra, we developed a multiband effective-mass model for gain dynamics in quantum-well diode structures. Transient photogeneration of electron-hole pairs by the pump pulse and subsequent relaxation of carriers by polar optical phonon and carrier-carrier scattering are investigated. Polar optical phonon scattering rates are described by a collision integral term in the Boltzmann equation and carrier-carrier scattering is modeled using an effective relaxation operator. The carrier-carrier scattering rates are determined using the quasiequilibrium distribution functions for a given background carrier density and the distribution function to which the photoexcited distribution relaxes is a Fermi-Dirac function where the chemical potential and temperature are selfconsistently chosen so that both particle number and energy are conserved in the carrier-carrier scattering process. The relaxation approximation for carrier-carrier scattering makes the problem an effective one-dimensional problem which can then be solved directly for the carrier distributions using an adaptive Runge-Kutta routine. This procedure is much less computationally intensive than a full Monte Carlo simulation. The results show that the inclusion of carrier-carrier scattering improves previous results where only carrierphonon scattering was included and that carrier-carrier scattering is necessary to produce heating of the carriers in the high-energy tails.

\section{ACKNOWLEDGMENTS}

We are grateful for comments and suggestions from Bjarne Tromborg, Jesper Mørk, and Gadi Eisenstein during our visit to Tele-Denmark Research. This work was supported by the U.S. Office of Naval Research through Grant No. N0001491-J-1956 and the Lincoln Laboratory Advanced Concept Program. C.J.S. gratefully acknowledges the assistance of the Alfred P. Sloan Foundation and the National Science Foundation Grant No. DMR 8957382 for summer support. We also wish to thank the Danish Research Academy for support during our visit to Denmark's Technical University in Lyngby.
*Permanent address: Department of Physics, University of Florida, Gainesville, FL 32611.

${ }^{1}$ B. C. Johnson and A. Mooradian, Appl. Phys. Lett. 49, 1135 (1986).

${ }^{2}$ J. M. Wiesenfeld, R. S. Tucker, and P. M. Downey, Appl. Phys. Lett. 51, 1307 (1987).

${ }^{3}$ J. M. Wiesenfeld, G. Eisnefeld, R. S. Tucker, G. Raybon, and P. B. Hansen, Appl. Phys. Lett. 53, 1239 (1988).

${ }^{4}$ G. Eisenstein, R. S. Tucker, J. M. Wiesenfeld, P. B. Hanson, G. Raybon, B. C. Johnson, T. J. Bridges, F. G. Stortz, and C. A. Burns, Appl. Phys. Lett. 54, 454 (1989).

${ }^{5}$ P. B. Hanson, J. M. Wiesenfeld, G. Eisenstein, R. S. Tucker, and G. Raybon, IEEE J. Quantum Electron. 25, 2611 (1989).
${ }^{6}$ G. Eisenstein, J. M. Wiesenfeld, M. Wegener, G. Sucha, D. S. Chemla, S. Weiss, G. Raybon, and U. Koren, Appl. Phys. Lett. 58, 158 (1991).

${ }^{7}$ M. S. Stix, M. P. Kesler, and E. P. Ippen, Appl. Phys. Lett. 48, 1722 (1986).

${ }^{8}$ M. P. Kesler and E. P. Ippen, Appl. Phys. Lett. 51, 1765 (1987).

${ }^{9}$ C. T. Hultgren, D. J. Dougherty, and E. P. Ippen, Appl. Phys. Lett. 61, 2767 (1992).

${ }^{10}$ K. L. Hall, J. Mark, E. P. Ippen, and G. Eisenstein, Appl. Phys. Lett. 56, 1740 (1990).

${ }^{11}$ Y. Lai, K. L. Hall, E. P. Ippen, and G. Eissenstein, IEEE Photon. Technol. Lett. 2, 711 (1990).

${ }^{12}$ K. L. Hall, Y. Lai, E. P. Ippen, G. Eisenstein, and U. Koren, 
Appl. Phys. Lett. 57, 2888 (1990).

${ }^{13}$ K. L. Hall, G. Lenz, E. P. Ippen, U. Koren, and G. Raybon, Appl. Phys. Lett. 61, 2512 (1992).

${ }^{14}$ C.-K. Sun, H. K. Choi, C. A. Wang, and J. G. Fujimoto, Appl. Phys. Lett. 62, 747 (1993).

${ }^{15}$ G. D. Sanders, C.-K. Sun, J. G. Fujimoto, H. K. Choi, C. A. Wang, and C. J. Stanton, Phys. Rev. B 50, 8539 (1994).

${ }^{16}$ C.-K. Sun, B. Golubovic, J. G. Fujimoto, H. K. Choi, and C. A. Wang, Opt. Lett. 20, 210 (1995).

${ }^{17}$ H. K. Choi and C. A. Wang, Appl. Phys. Lett. 57, 321 (1990).

${ }^{18}$ N. D. Wingreen, C. J. Stanton, and J. W. Wilkins, Phys. Rev.
Lett. 57, 1084 (1986).

${ }^{19}$ S. M. Goodnick and P. Lugli, Phys. Rev. B 37, 2578 (1988).

${ }^{20}$ G. D. Sanders and Y.-C. Chang, Phys. Rev. B 35, 1300 (1987).

${ }^{21}$ M. Asada, in Quantum Well Lasers, edited by Peter S. Zory, Jr. (Academic, New York, 1993).

22 J. L. Pankove, Optical Processes in Semiconductors (Dover, New York, 1975).

${ }^{23}$ C.-K. Sun, B. Golubovic, H. K. Choi, C. A. Wang, and J. G. Fujimoto, Appl. Phys. Lett. 66, 1650 (1995).

${ }^{24}$ C.-K. Sun, H. K. Choi, C. A. Wang, and J. G. Fujimoto, Appl. Phys. Lett. 63, 96 (1993). 